\title{
Nonlinear Dynamics of the Quadratic-Damping Helmholtz Oscillator
}

\author{
R. Fangnon, ${ }^{1}$ C. Ainamon, ${ }^{1}$ A. V. Monwanou, ${ }^{1}$ C. H. Miwadinou $\mathbb{D}^{1,2}$ and J. B. Chabi Orou ${ }^{1}$ \\ ${ }^{1}$ Laboratoire de Mécanique des Fluides de la Dynamique Nonlinéaire et de la Modélisation des Systèmes \\ Biologiques (LMFDNMSB), Institut de Mathématiques et de Sciences Physiques, Porto-Novo, Benin \\ ${ }^{2}$ Département de Physique, ENS-Natitingou, Université Nationale des Sciences Technologies, \\ Ingénierie et Mathématiques (UNSTIM), Abomey, Benin \\ Correspondence should be addressed to C. H. Miwadinou; clement.miwadinou@imsp-uac.org
}

Received 18 August 2020; Revised 14 October 2020; Accepted 23 October 2020; Published 9 November 2020

Academic Editor: Karthikeyan Rajagopal

Copyright (c) 2020 R. Fangnon et al. This is an open access article distributed under the Creative Commons Attribution License, which permits unrestricted use, distribution, and reproduction in any medium, provided the original work is properly cited.

\begin{abstract}
In this paper, the Helmholtz equation with quadratic damping themes is used for modeling the dynamics of a simple preypredator system also called a simple Lotka-Volterra system. From the Helmholtz equation with quadratic damping themes obtained after modeling, the equilibrium points have been found, and their stability has been analyzed. Subsequently, the harmonic oscillations have been studied by the harmonic balance method, and the phenomena of resonance and hysteresis are observed. The primary and secondary resonances have been researched by the multiple-scale method, and the conditions of stability of the amplitudes of oscillations are determined. Chaos is detected analytically by the Melnikov method and numerically using the basin of attraction, the bifurcation diagram, the Lyapunov exponent, the phase portrait, and the Poincare section. The effects of all the parameters of the system are analyzed in detail, and special emphasis is placed on the new parameters. Through this analysis, the complex phenomena such as hysteresis, bistability, amplitude jump, resonances, and chaos have been obtained. The control of the parameters and the necessary conditions to control the aforementioned phenomena have been found.
\end{abstract}

\section{Introduction}

Hermann von Helmholtz (1821-1894) is the origin of the very first acoustic resonator that bears his name. In order to extract the harmonics from total sound, he imagined a device made up of receptacles pierced with an orifice depending on their size and a frequency of resonance. To study the complexity of sounds and their perception by the human ear, he used resonators [1]. The dynamics of these resonators are governed by an oscillator equation called the Helmholtz oscillator. This oscillator presents analogies with traditional oscillators, namely, the mass-spring system, the simple pendulum, the circuit $(\mathrm{L}, \mathrm{C})$. As fields of application, these resonators are used in certain combustion boilers having concerns of acoustic transmission by the flue pipe in order to dissipate the phenomenon of resonance giving rise to vibrations in some boilers: boiler manufacturers use them to attenuate the noises coming out of the flue pipe. They are also used in cars, aeronautics, musical instruments, room acoustics, bass-reflex speakers, etc. This resonator, taking into account its multiple uses, was a center of interest for a number of researchers who competed with innovations and imaginations in order to solve physical problems on the basis of the functioning of this resonator. Indeed, Doelman et al. studied in [2] the irregularity of tidal oscillations due to the geometry of the basin from the following equation:

$$
\ddot{v}+\zeta(v)=\zeta_{\text {ext }}-\gamma(\dot{v})
$$

They have approached in their work a situation which generally has Helmholtz periods ranging from a few minutes to an hour. Balibrea et al. studied in [3] the stability of the structure of the Helmholtz oscillator under changes in the shape of nonlinear periodic disturbances. Komkin and Bykov in [4] had the characteristics of the Helmholtz resonator confirmed from experimental and analytical studies. 
In general, dynamic systems and nonlinear sciences constitute a large field of research, given their application in several fields: in mechanics, chemistry, quantum optics, astrophysics, hydrodynamics, electronics, biophysics, and so on [5-25]. Much of the discussion in the physics and engineering literature concerning damped oscillations, linear and nonlinear damping in certain applied systems play an important role since they may be used to suppress large amplitude oscillations or various instabilities, and they can also be used as a control mechanism [26-29]. For example, Soliman and Thompson in [27] and Miwadinou et al. in $[28,29]$ studied with considerable detail the effects of the damping level on the resonance response of the steady-state solutions and on the basin bifurcation patterns of the escape oscillator. In particular, they analyzed the effect of using different damping levels and how this contributes to the erosion of the safe areas in the phase space. On the contrary, nonlinear damping in vibrations has been investigated in [30-33]. In these different works, the authors studied the effects of nonlinear damping on the dynamics of viscoelastic plates, and interesting conclusions were drawn in relation to these effects on the performance of the viscoelastic plates used.

The Helmholtz oscillator is thus an example of a nonlinear dynamic system which is used to better understand nonlinear phenomena in acoustics, electronics, naval engineering naval, etc. [13-16]. It is in this order of ideas that we find a new model of the Helmholtz oscillator to better analyze the dynamics of a simple Lotka-Volterra system. From various previous works on the Helmholtz oscillator, we note that the new model of Helmholtz can be the seat of many phenomena. We can cite, for example, phenomena such as amplitude jump, hysteresis, nonlinear resonances, chaos, coexistence of attractors, and multistability [13-15]. The search for these phenomena for the new Helmholtz model therefore seems very important. The determination of the states of resonance in a nonlinear dynamic system makes it possible to predict energy exchanges by making the energy proportional to the square of the amplitude of the vibrations [18-21]. For example, in mechanical systems, a sudden increase in the resonance energy can cause damage to the mechanical system, while antiresonance systems can be used to store energy. Furthermore, the interest of nonlinear resonances for the new Helmholtz oscillator is great. Nowadays, most nonlinear vibration techniques focus on the method of modulation of upper harmonics and sidebands, but approaches to detect nonlinear damage based on nonlinear resonances require even more investigation. Thus, the condition giving the relation between the amplitude of vibration and the parameters of the system is crucial for the good choice of the frequency and the amplitude of excitation to trigger a nonlinear resonance effect [34-36]. The most suitable techniques for finding resonances are the harmonic balance and the multiple-scale methods [18, 19, 37]. The object of chaos theory is the study of nonlinear phenomena governed by simple and deterministic laws whose behavior under certain conditions becomes unpredictable. Since its discovery in the 20th century, chaos has been one of the most interesting for dynamic systems in areas such as physics, mathematics, chemistry, biochemistry, economics and finance, epidemiology, and engineering [19-25]. Depending on the field of study, it is sometimes useful or undesirable to the point where many researchers are interested in its prediction and/or control. One of the techniques used for the analytical determination of chaos is the Melnikov method. It is often used to research and predict horseshoe chaos [21, 26, 29].

In this paper, we study resonance, hysteresis, stability, and the chaos of a modified Helmholtz oscillator when it is subjected to an external periodic excitation. To do this, we consider the following system:

$$
\ddot{x}+x-x^{2}+\mu \dot{x}-\beta \dot{x}^{2}+\nu x \dot{x}=F \cos \Omega \tau,
$$

where $\mu, \nu$, and $\beta$, respectively, represent the linear, impure quadratic, and pure quadratic damping coefficients and $F$ and $\Omega$ are, respectively, the amplitude and the frequency of external excitation. One of the originalities here is the new model of the Helmholtz equation obtained by reducing the number of variables of the simple Lotka-Volterra system. The new themes are all quadratic damping themes. We determine the fixed points and find their stability and their nature for the autonomous system. We search the effect of the external force on the system by studying the resonances by the methods of the harmonic balance and the multiple scales. Melnikov's method is applied to the system to study its chaotic behavior. These different methods applied to the system allow us to evaluate the influence of various parameters of the system on the state of the system, to predict the variation of the maximum amplitude of the oscillations according to the parameters of the system, and to verify the existence of the phenomenon of jump and hysteresis in the system. The horseshoe chaos and dissipative chaos are also strongly studied and controlled. Analytical results are confirmed by numerical simulations.

The paper is structured as follows: Section 2 gives the mathematical modeling of a quadratic-damping Helmholtz oscillator and analyzes the fixed points and their stability. In Section 3, an in-depth detail of the harmonic oscillations is given. Section 4 deals with primary resonance and secondary resonance, while Section 5 analyzes the horseshoe chaos followed by the Melnikov analysis and the basin of attraction. In Section 6, the numerical analysis of the dissipative chaos is given in detail. Finally, we conclude the paper in Section 7 .

\section{Model, Fixed Points, and Stability}

In this work, we consider the following simple Lotka-Volterra system [12]:

$$
\begin{gathered}
\dot{\xi}=K_{\xi} a \xi-K_{\eta} \xi \eta \\
\dot{\eta}=K_{\eta} \xi \eta-K_{d} \eta
\end{gathered}
$$

where $\xi$ represents the population of rabbits reproducing in an autocatalytic manner, $\eta$ is the lynx population, $K_{d}$ is the lynx mortality rate, $K_{\eta}$ provides information on how fast lynxes breed, $K_{\xi}$ is a speed constant which is the rabbit's 


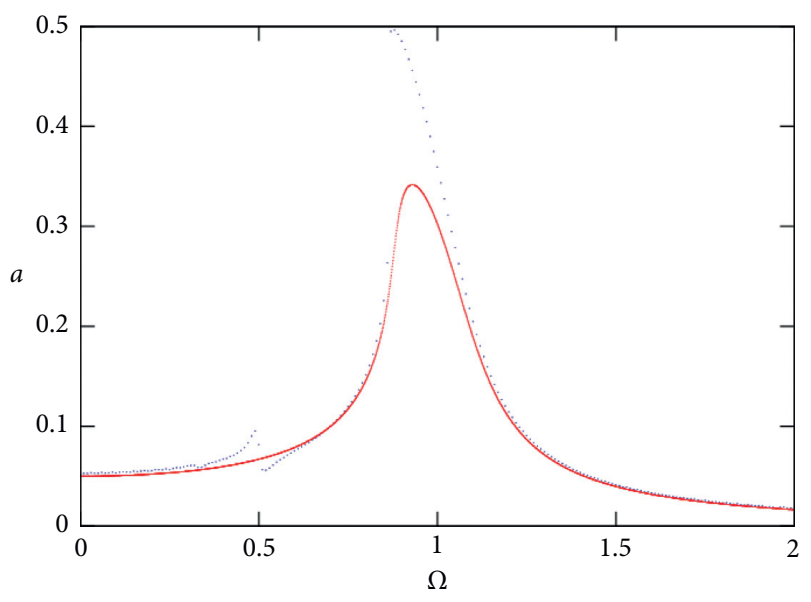

- Numerical result

— Analytical result

FIGURE 1: Comparison between analytical and numerical frequency-response curve $a(\Omega)$ with the parameters $\beta=0.05, \nu=1.75$, $\mu=0.05$, and $F=0.05$.

reproduction speed, and $a$ is the quantity of grass assumed to be constant. Setting $K_{\xi} a=\alpha, K_{\eta}=\lambda$, and $K_{d}=\gamma$, equation (3) becomes

$$
\begin{gathered}
\dot{\xi}=\alpha \xi-\lambda \xi \eta, \\
\dot{\eta}=-\gamma \eta+\lambda \xi \eta .
\end{gathered}
$$

In order to simplify the calculations, we define $x=\lambda \xi+\lambda \eta$, and we find

$$
\begin{gathered}
\xi=\frac{\dot{x}+\gamma x}{\lambda(\gamma+\alpha)}, \\
\eta=\frac{-\dot{x}+\alpha x}{\lambda(\gamma+\alpha)} .
\end{gathered}
$$

Replacing equation (5) in equation (4), we obtain

$$
\ddot{x}+\omega_{0}^{2} x-\bar{\delta} x^{2}+\bar{\mu} \dot{x}-\beta \dot{x}^{2}+\bar{v} x \dot{x}=0,
$$

with $\quad-\omega_{0}^{2}=\alpha \gamma, \bar{\delta}=-\alpha \gamma /(\alpha+\gamma), \beta=1 /(\alpha+\gamma), \bar{\mu}=\gamma-$ $\alpha$, and $\bar{\nu}=(\alpha-\gamma) / \alpha+\gamma$.

When the system is under external periodic excitation, equation (6) becomes

$$
\ddot{x}+\omega_{0}^{2} x-\bar{\delta} x^{2}+\bar{\mu} \dot{x}-\beta \dot{x}^{2}+\bar{v} x \dot{x}=F \cos \omega t .
$$

Now, setting $\Omega=\left(\omega / \omega_{0}\right)$ and $\tau=\omega_{0} t$, one has

$$
\ddot{x}+x-\delta x^{2}+\mu \dot{x}-\beta \dot{x}^{2}+\nu x \dot{x}=F \cos \Omega \tau,
$$

with $\delta=\left(\bar{\delta} / \omega_{0}^{2}\right), \mu=\left(\bar{\mu} / \omega_{0}\right)$, and $\nu=\left(\bar{\nu} / \omega_{0}\right)$.

$F$ and $\Omega$ designate, respectively, the amplitude and the frequency of the external periodic excitation; $\mu, \beta$, and $\nu$ are real parameters of the system. Equation (8) represents the equation of a Helmholtz forced oscillator with pure quadratic damping $\left(\beta \dot{x}^{2}\right)$ and hybrid damping $(\nu x \dot{x})$. When $\beta$ and $\nu$ are all zero, equation (8) is reduced to the equation of a Helmholtz oscillator $[9,10,14]$. The Helmholtz oscillator is widely used in several areas to study the dynamics of certain systems. Indeed, in naval architects, the Helmholtz oscillator, known as the Helmholtz-Thompson equation, is used to analyze the escape phenomenon. A detailed dynamical analysis of the Helmholtz oscillator is done by Thompson in [9], and Gottwald et al. experimentally studied in [16] the dynamics of this oscillator. Helmholtz-Thompson equation finds direct application in the investigation of the bubble dynamics [17] and is much discussed in the naval architecture literature (see [13]). These concepts continue to find fruitful applications in quantification of capsize resistance [15].

In the remaining of this section, we determine the fixed points of the autonomous Helmholtz oscillator with quadratic damping and study their stability. Indeed, taking $\delta=1$, the autonomous system is in the following form:

$$
\begin{array}{r}
\dot{x}=y, \\
\dot{y}=-x+x^{2}-\mu y+\beta y^{2}-\nu x y .
\end{array}
$$

Setting $\dot{x}=0$ and $\dot{y}=0$, the equilibrium points of (9) are $A_{0}(0,0)$ and $A_{1}(1,0)$. The eigenvalues $\lambda$ associated with the fixed point $A_{0}$ verify

$$
\lambda^{2}+\mu \lambda+1=0
$$

As a result,

(i) If $\mu^{2} \geq 4$, then $\lambda_{1}=-\mu-\sqrt{\mu^{2}-4} / 2$ and $\lambda_{1}=-\mu+\sqrt{\mu^{2}-4} / 2$.

(a) $\mu \leq-2$, the eigenvalues $\lambda_{1}$ and $\lambda_{2}$ are positive. $A_{0}$ is an unstable repulsive node.

(b) $\mu \leq 2$, the eigenvalues $\lambda_{1}$ and $\lambda_{2}$ are negative. $A_{0}$ is a stable attractive node.

(ii) If $\mu^{2}<4$, then $\lambda_{1}=-\mu-i \sqrt{\mu^{2}-4} / 2$ and $\lambda_{2}=-\mu+i \sqrt{\mu^{2}-4} / 2$.

(a) $-2<\mu<0$, the eigenvalues $\lambda_{1}$ and $\lambda_{2}$ are complexes whose real parts are positive. $A_{0}$ is an unstable repulsive focus.

(b) $0<\mu<2$, the eigenvalues $\lambda_{1}$ and $\lambda_{2}$ are complexes whose real parts are negative. $A_{0}$ is a stable attractive focus.

Now, the eigenvalues $\lambda$ associated with the fixed point $A_{1}$ are solutions of

$$
\lambda^{2}+(\mu+\nu) \lambda-1=0 .
$$

For all values of $\mu$ and $\nu$ one has $\lambda_{1}=-(\mu+\nu)-\sqrt{(\mu+\nu)^{2}+4} / 2 \quad$ and $\lambda_{2}=-(\mu+\nu)+\sqrt{(\mu+\nu)^{2}+4 / 2}$. It is easy to note that these eigenvalues are of opposite signs, and therefore, $A_{1}$ is a saddle point.

\section{Harmonic Oscillations}

In this part, we use the harmonic balance method to study harmonic oscillations. For this, we take the solutions of equation (8) in the following form $[18,19,37]$ : 


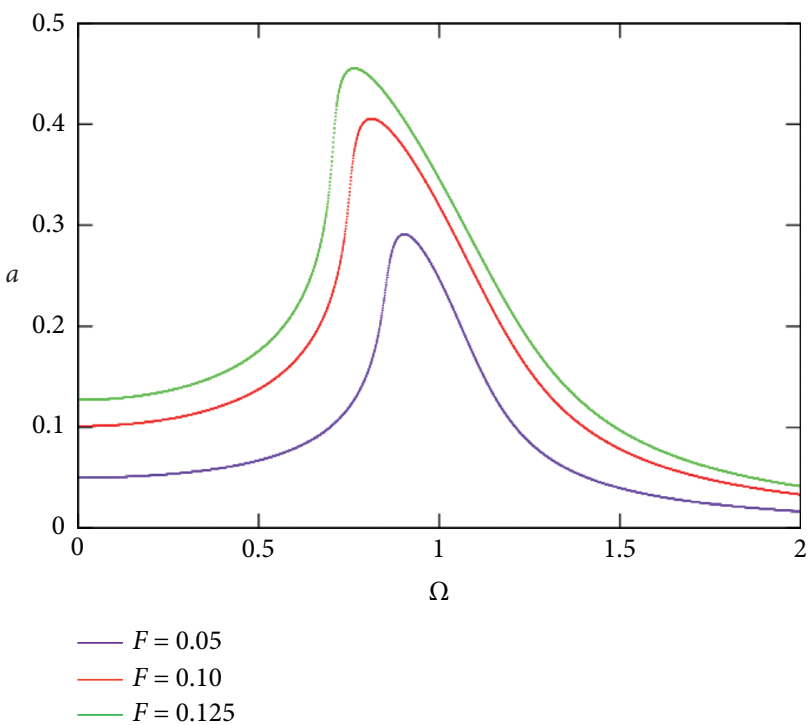

(a)

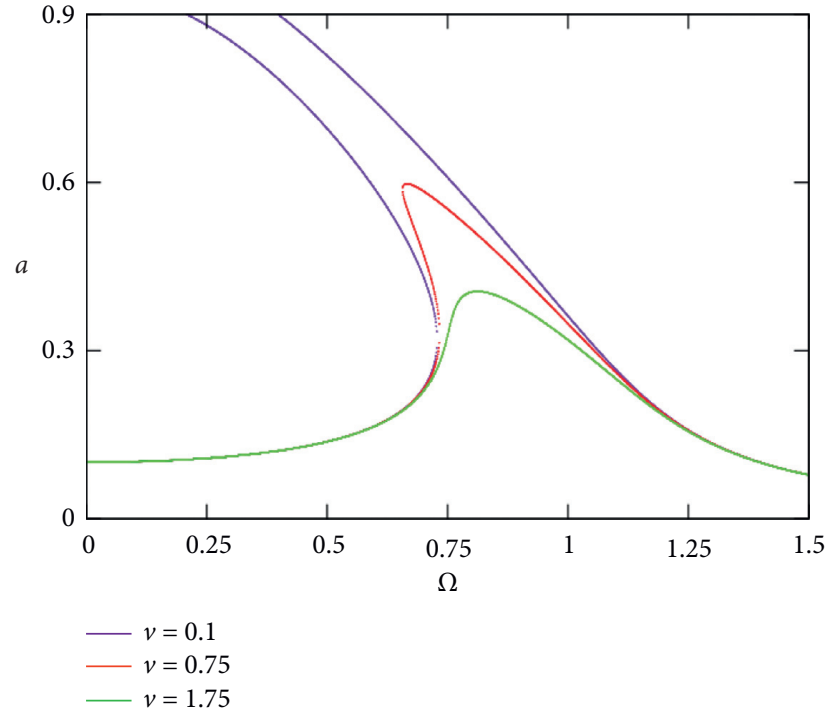

(b)

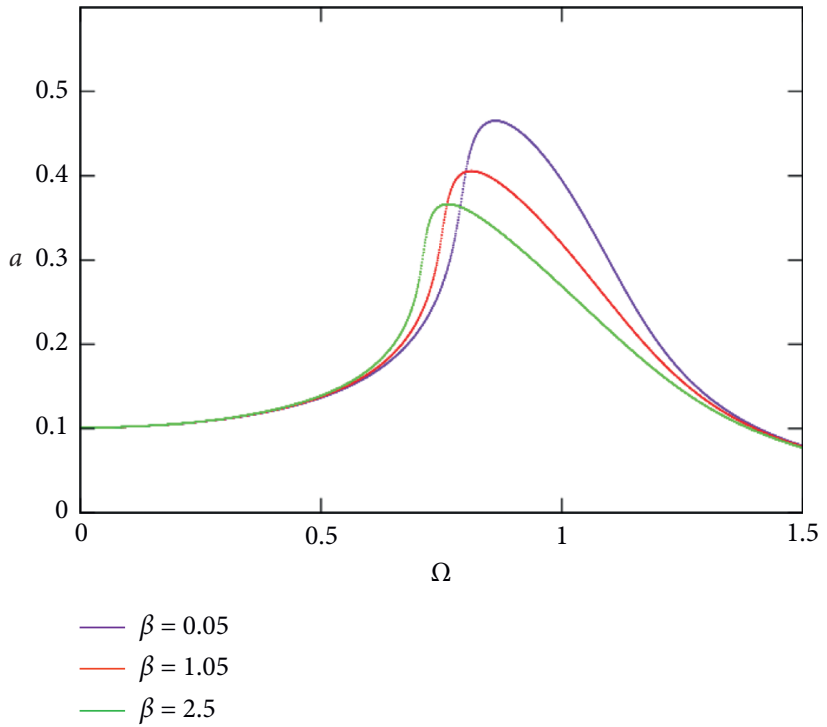

(c)

FIGURE 2: Variation curve of the amplitude $a$ as a function of the excitation frequency with $\mu=0.05$ : (a) effect of $F$, (b) effect of $\nu$, and (c) effect of $\beta$.

$$
x=a \cos (\Omega \tau+\phi),
$$

with $|J| \ll|a|$ and $J^{n}=0$ for $(n \geq 2)$. By inserting equation (12) into equation (8), after some mathematical manipulations, we obtain the following expression verified by the amplitude of the harmonic oscillations:

$$
\left[\mu a \Omega+\frac{1}{2} \nu \Omega a^{3}\left(\beta \Omega^{2}+1\right)\right]^{2}+\left[-a+a^{3}\left(\beta \Omega^{2}+1\right)+a \Omega^{2}\right]^{2}=F^{2}
$$

Before studying the influence of each parameter on the amplitude of the oscillations obtained, we compared the analytical result (equation (13)) to the numerical result by representing in Figure 1 the amplitude $a$ as a function of the external frequency $\Omega$. The basic values of the parameters used here and throughout this work are those used in the literature $[9,14]$. We note a coherence between these two results which shows that the system has a nonlinear resonance. Figure 2 shows the effects of the amplitude $F$ of the external force (Figure 2(a)), the coefficient $v$ of the hybrid quadratic damping term (Figure 2(b)), and the coefficient $\beta$ of the pure quadratic damping term (Figure 2(c)). Through these figures, we note the presence of stable and unstable amplitudes whose domains of existence are strongly influenced by $F, \beta$, and $\nu$. We also note that the amplitude and the frequency of the resonance and the bandwidth lag are strongly influenced by these parameters. Precisely, the amplitude of the response increases with $F$ unlike $\beta$ and $\nu$. The resonance frequency decreases with the increase of the 


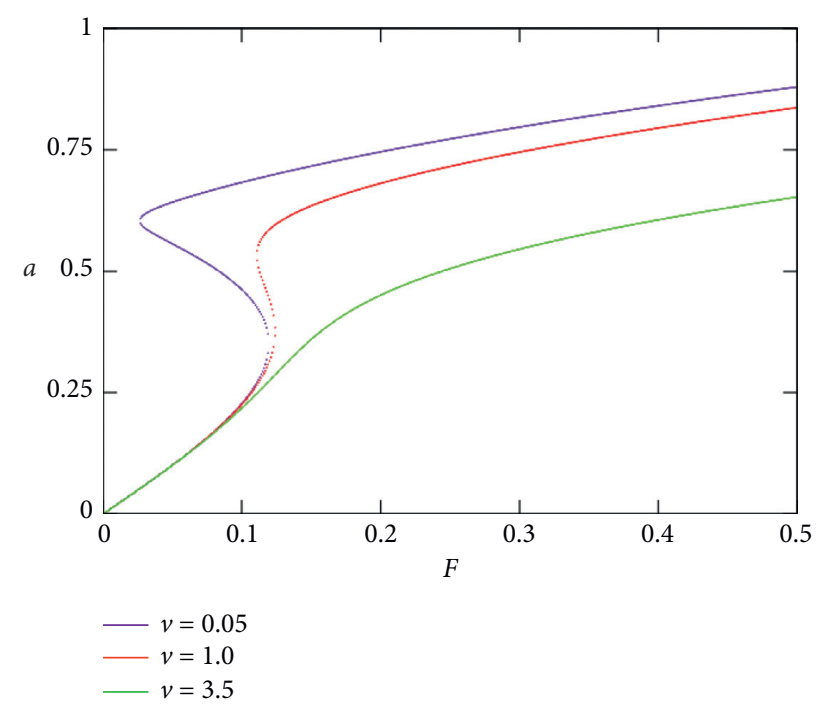

(a)

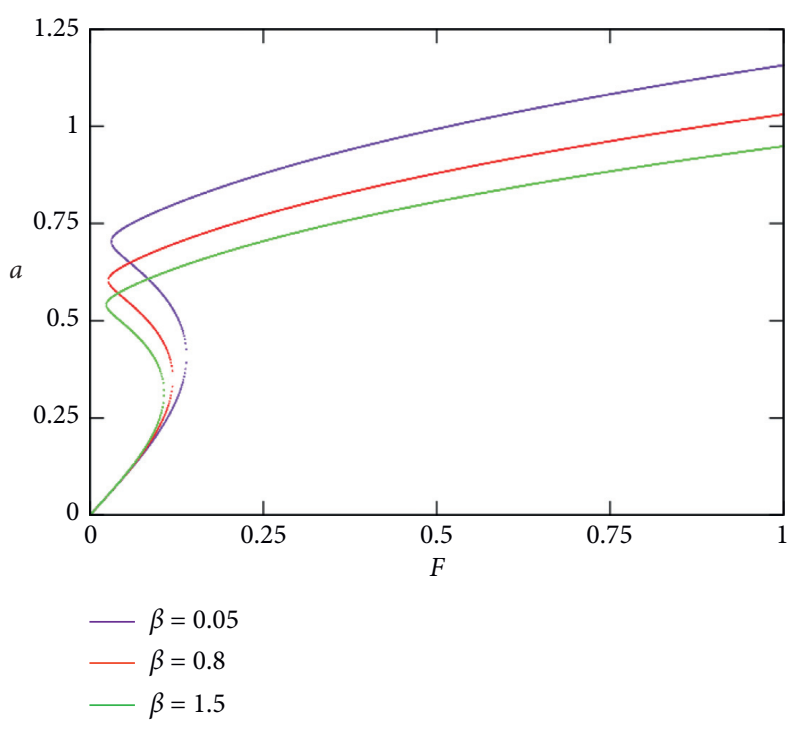

(b)

Figure 3: Hysteresis curve giving the variation of a function of $F$ with $\mu=0.05$ and $\Omega=0.7$ : (a) effect of $\nu$ and (b) effect of $\beta$.

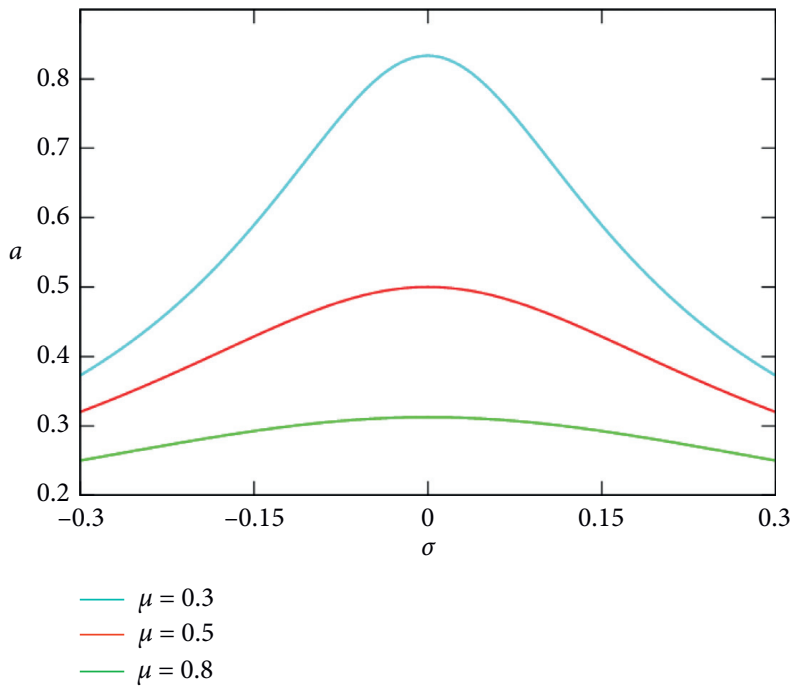

Figure 4: Curve of the variation of $a$ as a function of $\sigma$ for the primary resonance.

parameters $F$ and $\beta$, while it increases with $\nu$. We also notice through Figure 2(b) that the resonance is achieved at infinity if $\nu \leq 0.1$ for fixed values of $F$ and $\beta$. On the contrary, a system which undergoes a phenomenon of hysteresis can be considered as a memory system. This application is widely explored in engineering and technology. It is for this reason that the hysteresis and the amplitude jump are studied. Indeed, Figure 3 gives the variation of the amplitude $a$ of the oscillations according to the amplitude $F$ of the external excitation. From this figure, the phenomena of hysteresis and amplitude jump are observed and are influenced by the parameters of the system of which the most important are those of $v$ and $\beta$. During amplitude jump and hysteresis phenomenon processes, for any values of external excitation $F$, three different amplitudes of oscillations are obtained, among which two are stable and one is unstable (see the curve in red in Figure 3(a), for example). Also, note that the parameter $v$ can facilitate the appearance as well as the disappearance of each of these two phenomena and therefore will allow them to be controlled. In short, the harmonic oscillations of the system can be accompanied by phenomena of nonlinear resonance, amplitude jump, and hysteresis which are very common and are searched for in memory systems.

\section{Primary and Secondary Resonances}

In order to analyze the primary and secondary resonances, we use the multiple time-scale method; the solution of equation (8) can be expressed in the following form [18-20]:

$$
x(\tau, \varepsilon)=x_{0}\left(T_{0}, T_{1}\right)+\varepsilon x_{1}\left(T_{0}, T_{1}\right)+\cdots,
$$




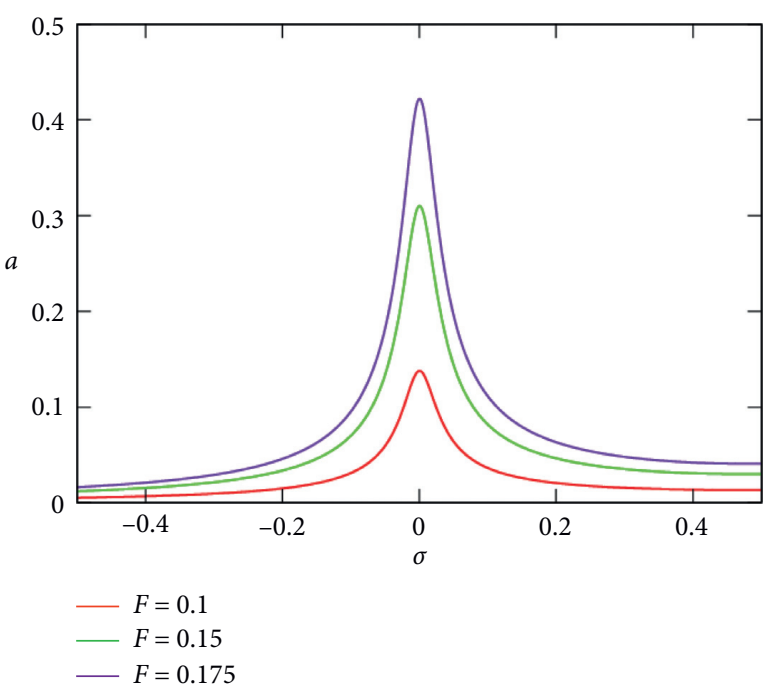

(a)

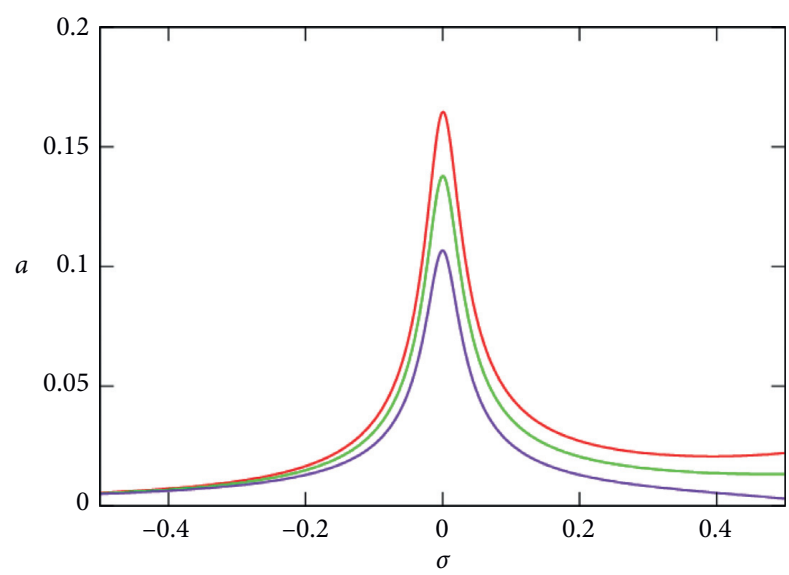

$-\beta=0.2$

$-\beta=0.8$

$-\beta=1.5$

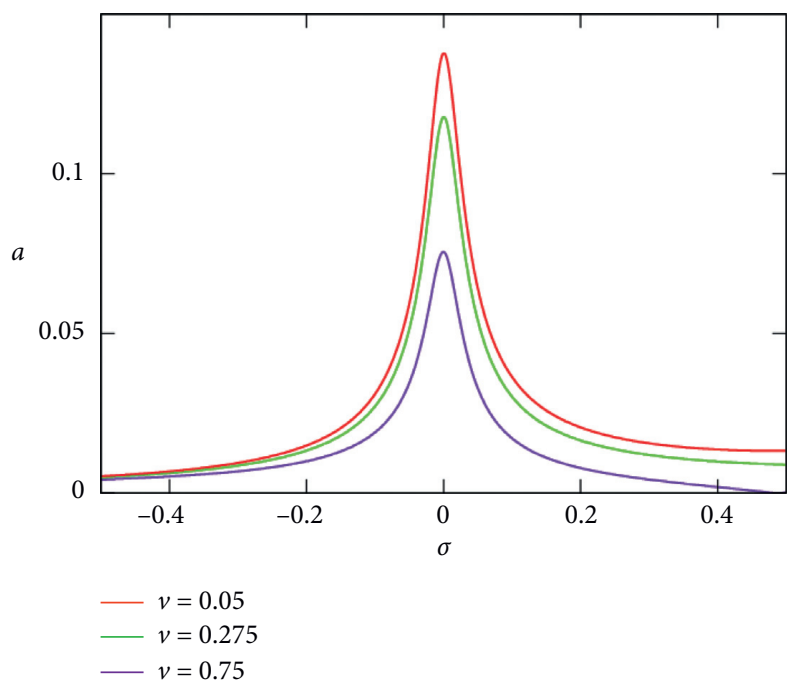

(c)

FIGURE 5: Curve of the variation of $a$ as a function of $\sigma$ for superharmonic order resonance 2 with $\mu=0.05$ : (a) effect of $F$, (b) effect of $\beta$, and (c) effect of $v$.

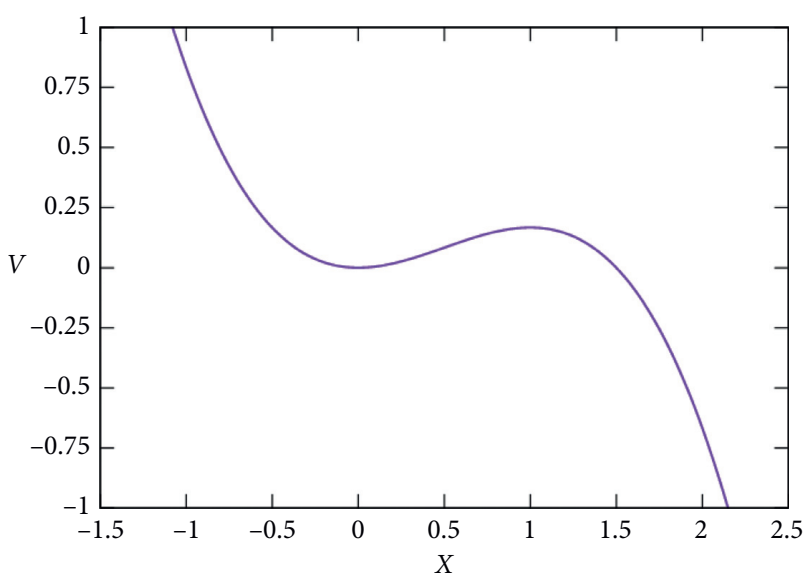

(a)

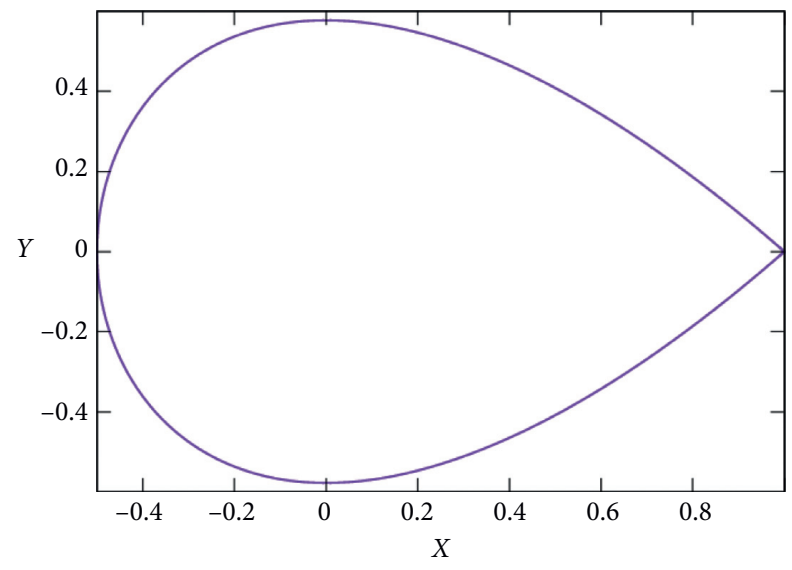

(b)

Figure 6: (a) Potential $V(x)$ and (b) homoclinic orbits. 


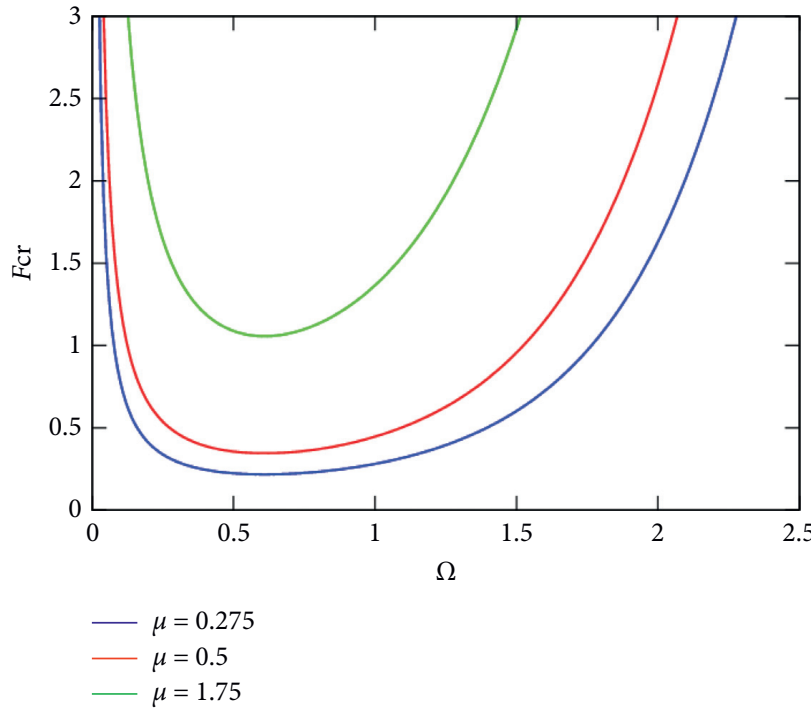

(a)

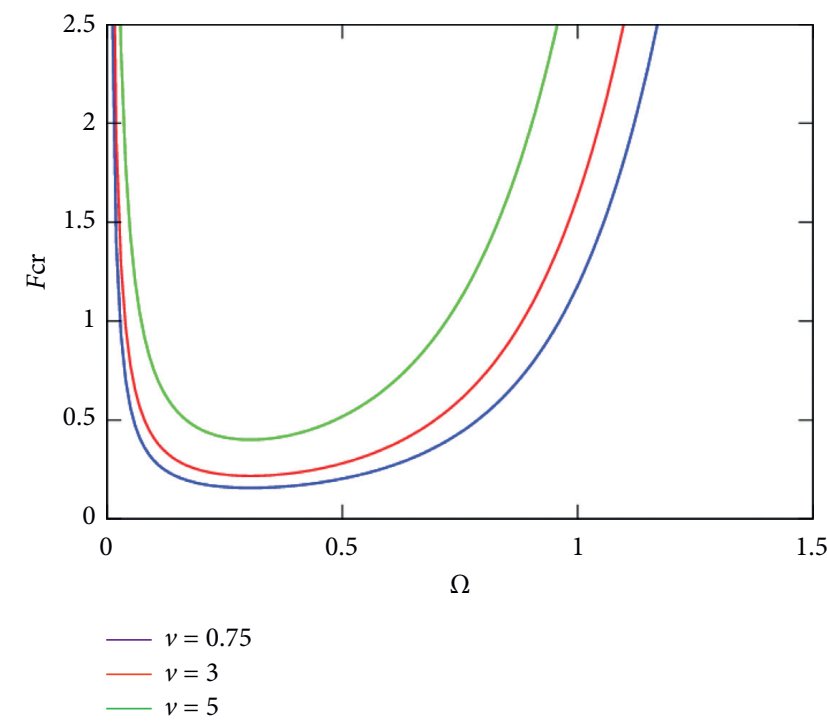

(b)

Figure 7: Variation curve of $F_{\text {cr }}$ as a function of $\Omega$ for the Melnikov chaos: (a) effect of $\mu$ and (b) effect of $\nu$.

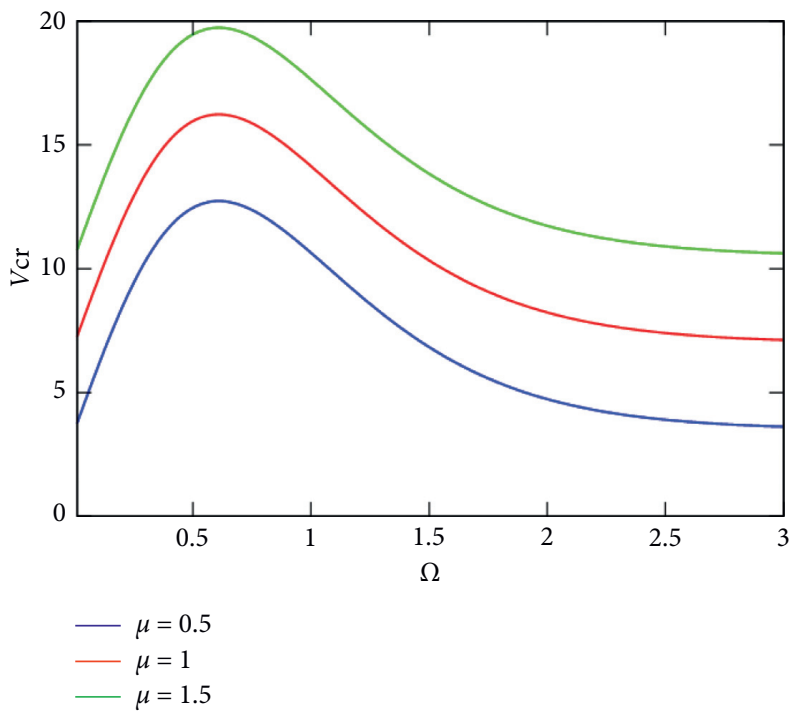

(a)

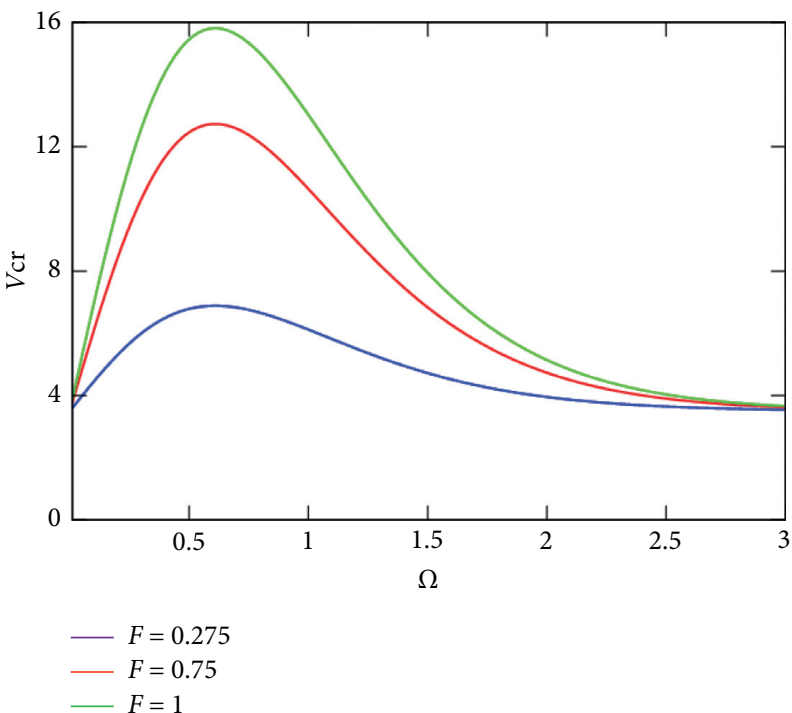

(b)

Figure 8: Variation curve of $\nu_{c r}$ as a function of $\Omega$ for the Melnikov chaos: (a) effect of $\mu$ and (b) effect of F.

where $T_{0}=\tau, T_{1}=\varepsilon \tau$, and $\varepsilon$ is the small pertubative parameter. The time derivatives transform into

$$
\begin{gathered}
\frac{\mathrm{d}}{\mathrm{d} \tau}=D_{0}+\varepsilon D_{1}+\cdots, \\
\frac{\mathrm{d}^{2}}{\mathrm{~d} \tau^{2}}=D_{0}^{2}+2 \varepsilon D_{0} D_{1}+\cdots,
\end{gathered}
$$

where $D_{0} \equiv \partial / \partial T_{0}$ and $D_{1} \equiv \partial / \partial T_{1}$.
4.1. Primary Resonance. To search for the primary resonance, we disturb all the nonlinear terms and that of the external excitation, and we use formulas (14)-(16). We obtain

$$
\begin{aligned}
D_{0}^{2} x_{0} & +\varepsilon\left(D_{0}^{2} x_{1}+2 D_{0} D_{1} x_{0}\right)+x_{0}+\varepsilon x_{1}-\varepsilon x_{0}^{2} \\
& +\varepsilon \mu D_{0} x_{0}-\varepsilon \beta\left(D_{0} x_{0}\right)^{2}+\varepsilon \nu x_{0} D_{0} x_{0}=\varepsilon F \cos \Omega \tau
\end{aligned}
$$

By identification, we get the following. For $\varepsilon^{0}$, 


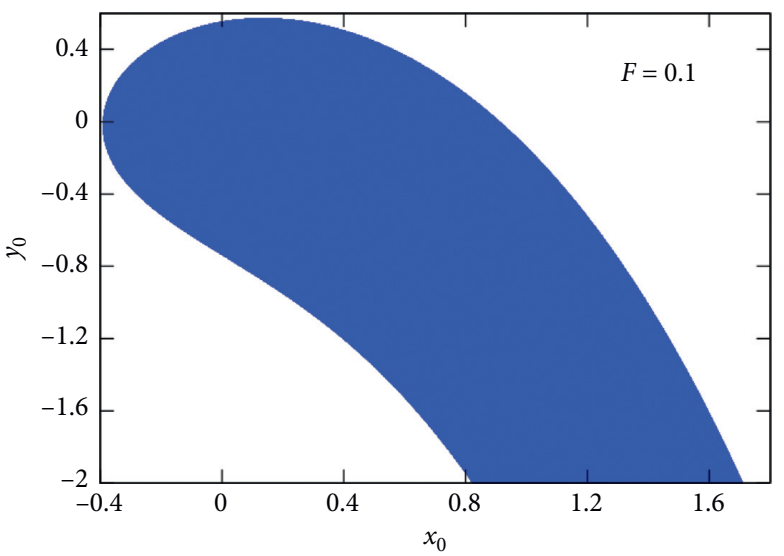

(a)

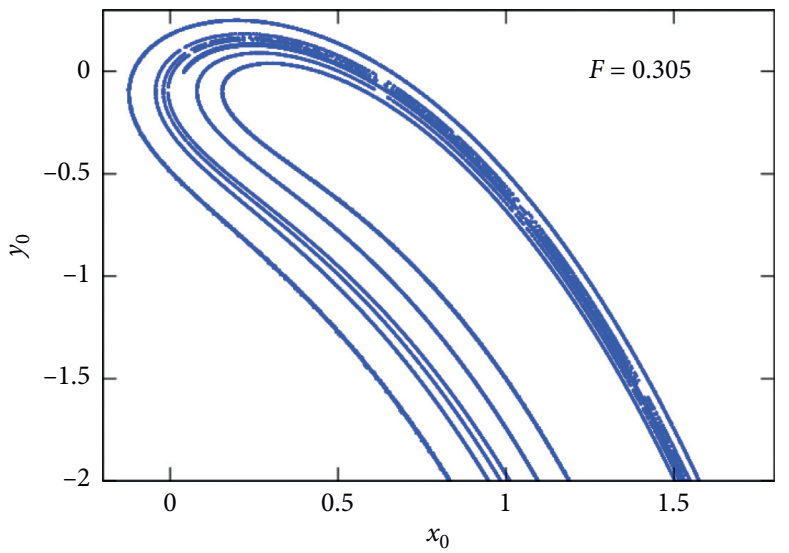

(c)

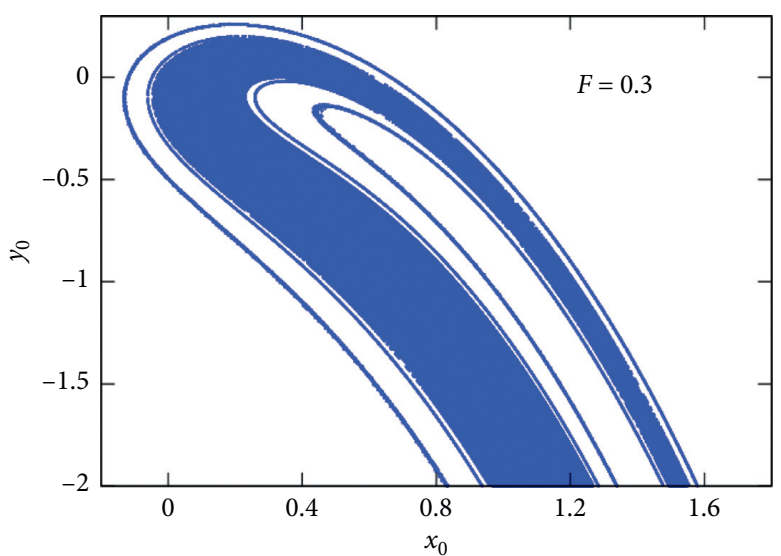

(b)

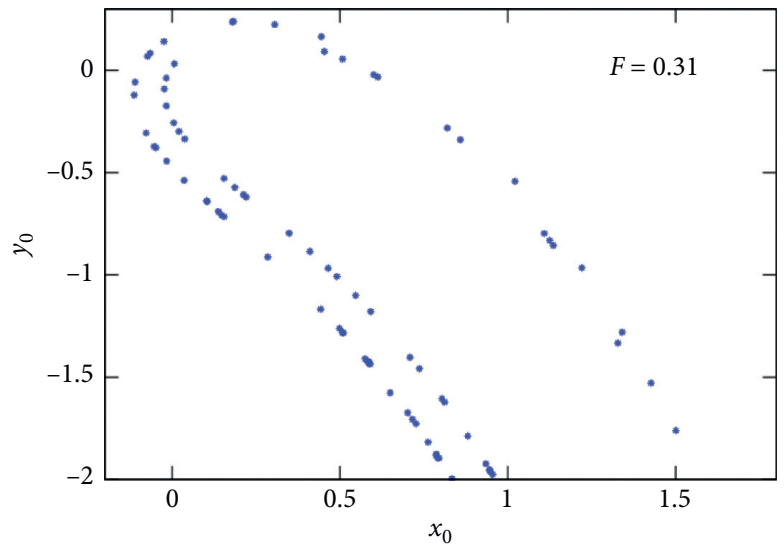

(d)

Figure 9: Effect of $F$ on the basin of attraction with $\Omega=0.297$ and other parameters of Figure 7 .

$$
D_{0}^{2} x_{0}+x_{0}=0
$$

For $\varepsilon^{1}$

$$
\begin{aligned}
D_{0}^{2} x_{1}+x_{1}= & -2 D_{0} D_{1} x_{0}+x_{0}^{2}-\mu D_{0} x_{0}-\beta\left(D_{0} x_{0}\right)^{2} \\
& -\nu x_{0} D_{0} x_{0}+F \cos \Omega \tau .
\end{aligned}
$$

A solution of equation (18) is

$$
x_{0}=A\left(T_{1}\right) e^{j T_{0}}+A^{*}\left(T_{1}\right) e^{-j T_{0}} .
$$

Inserting equation (20) into equation (19), we obtain

$$
\left(D_{0} x_{0}\right)^{2}=2 A A^{*}-A^{2}\left(T_{1}\right) e^{2 j T_{0}}+A^{* 2}\left(T_{1}\right) e^{-2 j T_{0}} .
$$

The primary resonance appears when $\Omega=1+\varepsilon \sigma, \sigma$ is the detuning parameter. So, we have

$$
D_{0}^{2} x_{1}+x_{1}=-2 j \frac{\mathrm{d} A}{\mathrm{~d} T_{1}}-j \mu A-\frac{F}{2} e^{j \sigma T_{1}}=0 .
$$

We take the polar form of $A$ :

$$
A=\frac{a}{2} e^{j \theta},
$$

and we put in equation (22). After some mathematical transformations, we obtain

$$
\begin{gathered}
a \sigma-a b^{\prime}=\frac{F}{2} \cos b, \\
-a^{\prime}-\frac{1}{2} \mu a=\frac{F}{2} \sin b,
\end{gathered}
$$

where $b=\sigma T_{1}-\theta$. By searching for steady-state solutions, we get

$$
(a \sigma)^{2}+\left(\frac{1}{2} \mu a\right)^{2}=\left(\frac{F}{2}\right)^{2}
$$

Equation (26) represents the primary resonance equation. Let us now study the stability of the amplitudes of oscillations by posing $a=a_{0}+a_{1}$ and $b=b_{0}+b_{1}$, where $\left(a_{0}\right.$, $\left.b_{0}\right)$ is the nontrivial solution and $\left(a_{1}, b_{1}\right)$ is assumed to be infinite. So,

$$
\begin{array}{r}
a_{1}^{\prime}=-\frac{1}{2} \mu a_{1}-\frac{F}{2} b_{1} \cos b_{0}, \\
b_{1}^{\prime}=\frac{F}{2 a_{0}^{2}} a_{1} \cos b_{0}+\frac{F}{2 a_{0}} b_{1} \sin b_{0} .
\end{array}
$$

It follows from the calculations that the amplitudes of oscillations are stable if $\mu>0$ and $\sigma^{2}+\left(\mu^{2} / 4\right)>0$. Either the amplitudes of the oscillations are stable for $\mu>0$ and 


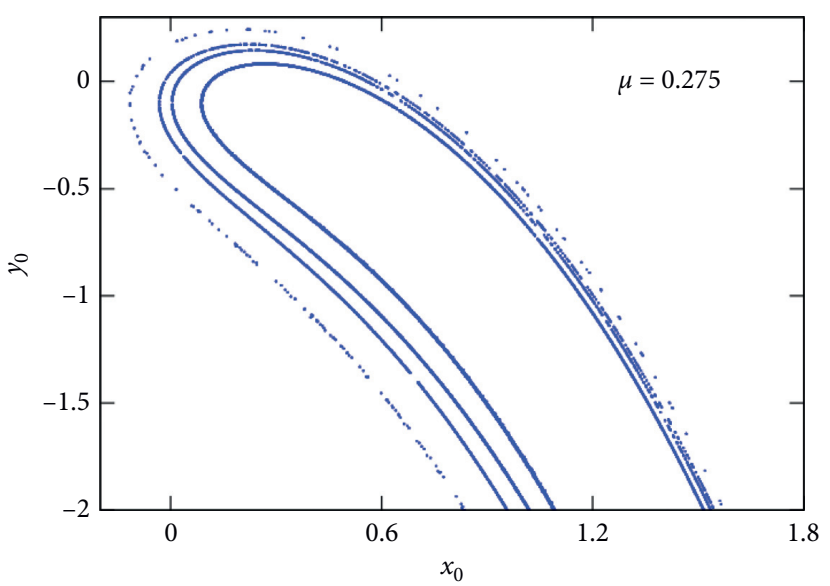

(a)

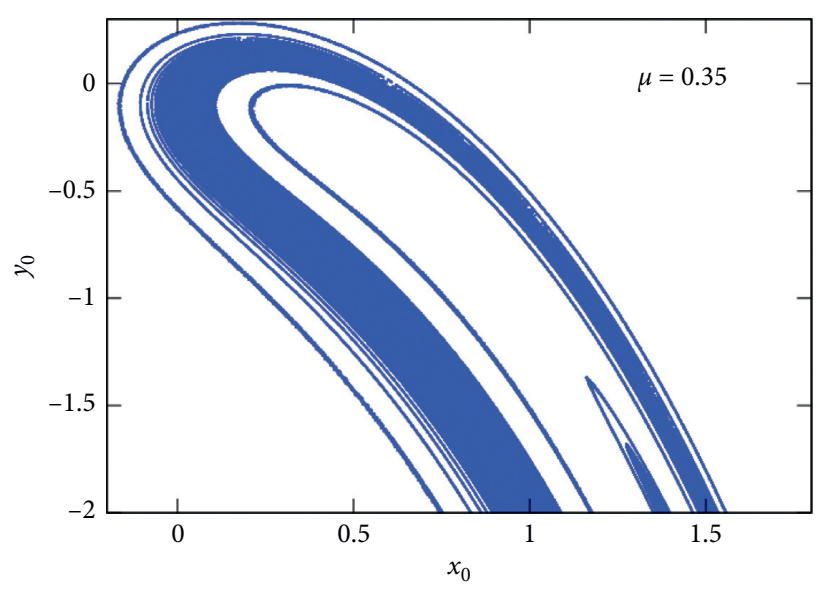

(b)

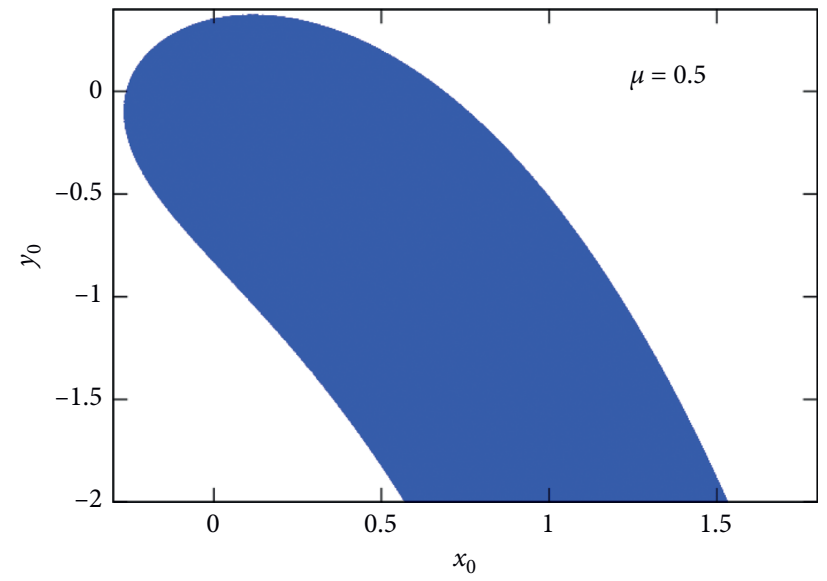

(c)

FIGURE 10: Effect of $\mu$ on the basin of attraction with $\Omega=0.297, F=0.308$, and other parameters of Figure 7 .

unstable if $\mu<0$. Figure 4 shows that when the linear dissipation increases, the amplitude of the response decreases considerably, and the resonance disappears.

4.2. Secondary Resonance. For this case, all the terms of nonlinearity are disturbed apart from the term of external force. So, using formulas (14)-(16), we get the following.

For $\varepsilon^{0}$,

$$
D_{0}^{2} x_{0}+x_{0}=F \cos \Omega \tau
$$

For $\varepsilon^{1}$

$D_{0}^{2} x_{1}+x_{1}=-2 D_{0} D_{1} x_{0}+x_{0}^{2}-\mu D_{0} x_{0}-\beta\left(D_{0} x_{0}\right)^{2}-\nu x_{0} D_{0} x_{0}$.

A solution of equation (28) is

$$
x_{0}=A\left(T_{1}\right) e^{j T_{0}}+A^{*}\left(T_{1}\right) e^{-j T_{0}}+B e^{j \Omega T_{0}}+B e^{-j \Omega T_{0}},
$$

with

$$
B=\frac{F}{2\left(1-\Omega^{2}\right)}
$$

Replacing equation (30) in equation (29), one has

$$
\begin{aligned}
& 2 j \frac{\mathrm{d} A}{\mathrm{~d} T_{1}} e^{j T_{0}}+j \mu A e^{j T_{0}}+\left(-B^{2}+\beta B^{2} \Omega^{2}+\nu B^{2} \Omega\right) e^{2 j \Omega T_{0}} \\
& +\left(-2 A^{*} B-\beta \Omega A^{*} \Omega+\nu A^{*} B\right) e^{j(\Omega-1) T_{0}}+\text { NST }+ \text { c.c. }=0,
\end{aligned}
$$

where NST denotes the nonsecular terms and c.c. denotes the conjugate complexes. By analyzing equation (32), we note that the system has a superharmonic resonance of order 2 and a subharmonic resonance of order 2. After studying, we noted that the superharmonic resonance of order 2 is important and therefore is the only one presented in this work.

Indeed, there is a superharmonic resonance if $2 \Omega=1+\varepsilon \sigma$. After treatment of equation (32), by considering the secular terms, we obtain

$$
2 j \frac{\mathrm{d} A}{\mathrm{~d} T_{1}} e^{j T_{0}}+j \mu A e^{j T_{0}}+\left(-B^{2}+\beta B^{2} \Omega^{2}+\nu B^{2} \Omega\right) e^{j(1+\varepsilon \sigma) T_{0}} .
$$

By inserting the polar form of $A$, 


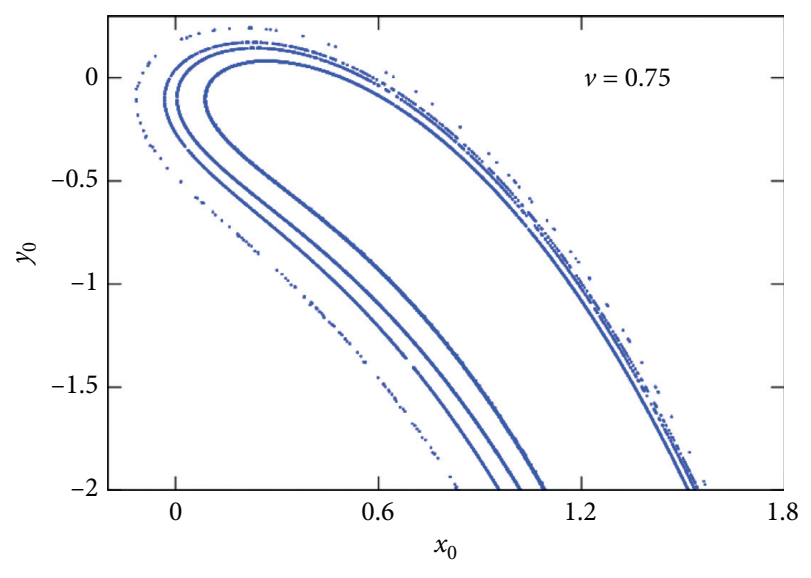

(a)

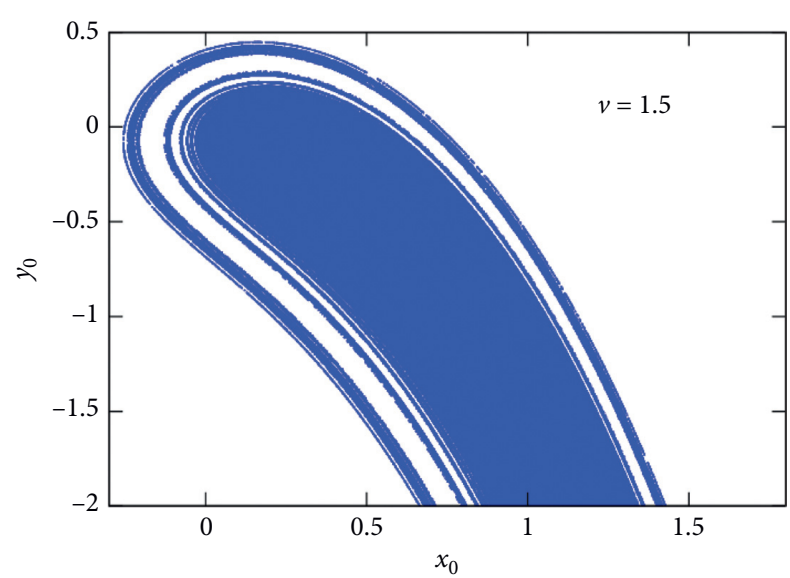

(b)

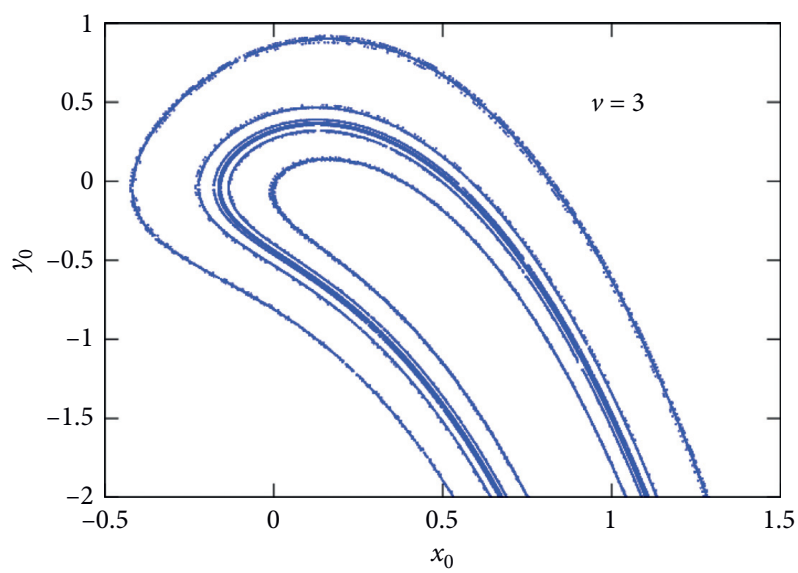

(c)

FIGURE 11: Effect of $\nu$ on the basin of attraction with $\Omega=0.297, F=0.308$, and other parameters of Figure 7 .

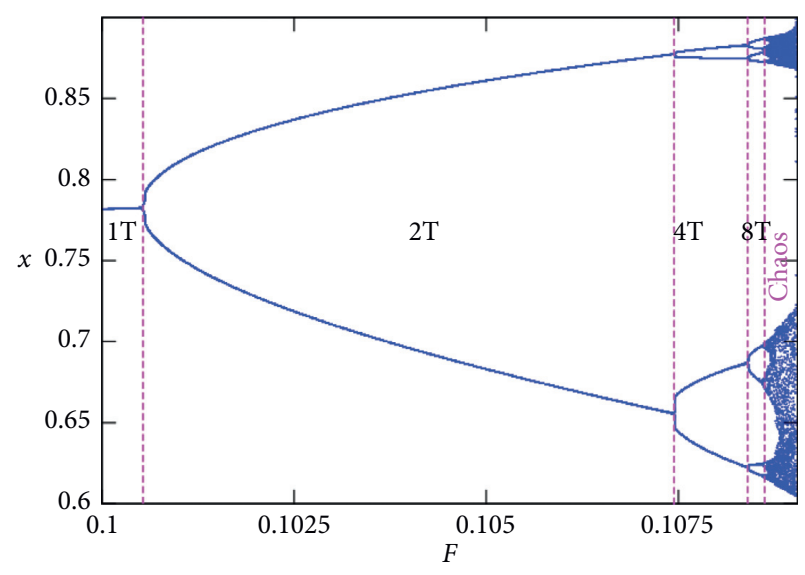

(a)

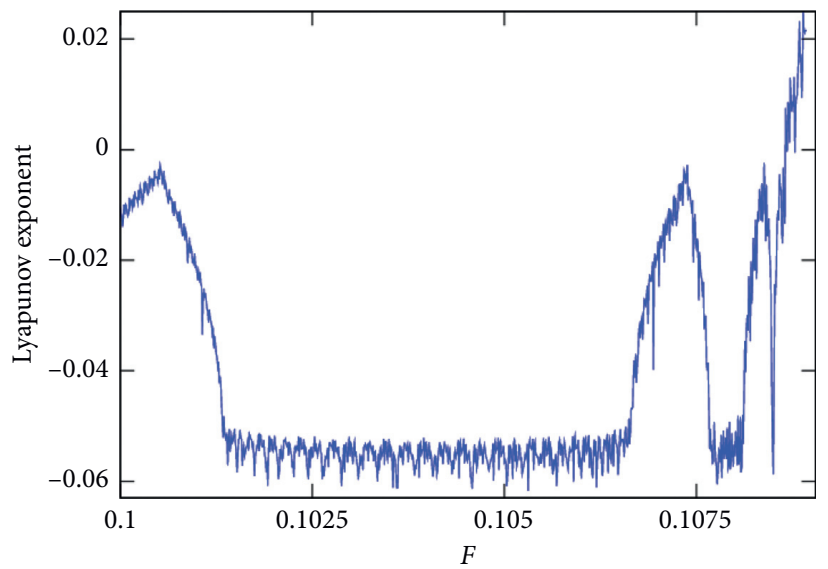

(b)

FIGURE 12: Bifurcation diagram and its corresponding Lyapunov exponent curve of a quadratic-damping Helmholtz oscillator versus $F$ with $\mu=0.01, \Omega=0.85$, and $\nu=\beta=0$.

$$
A=\frac{a}{2} e^{j \theta\left(T_{1}\right)}
$$

in equation (33) and by canceling the real and imaginary parts, we have 


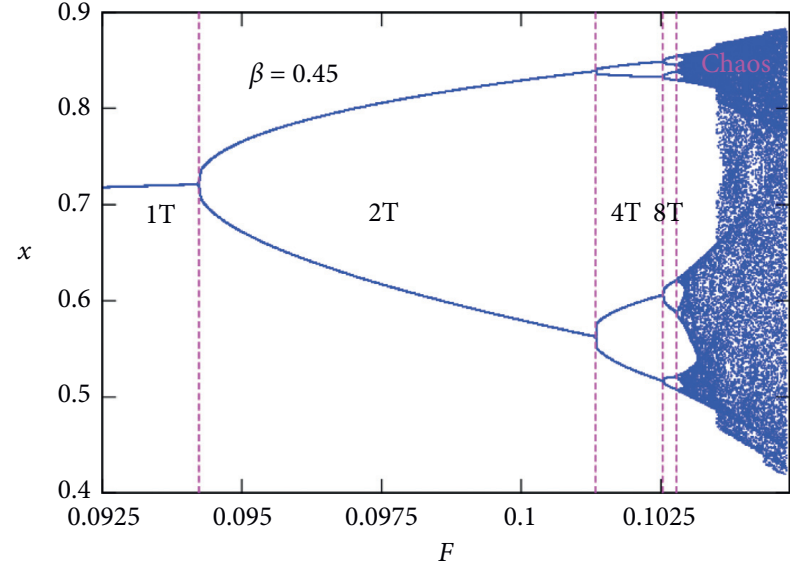

(a)

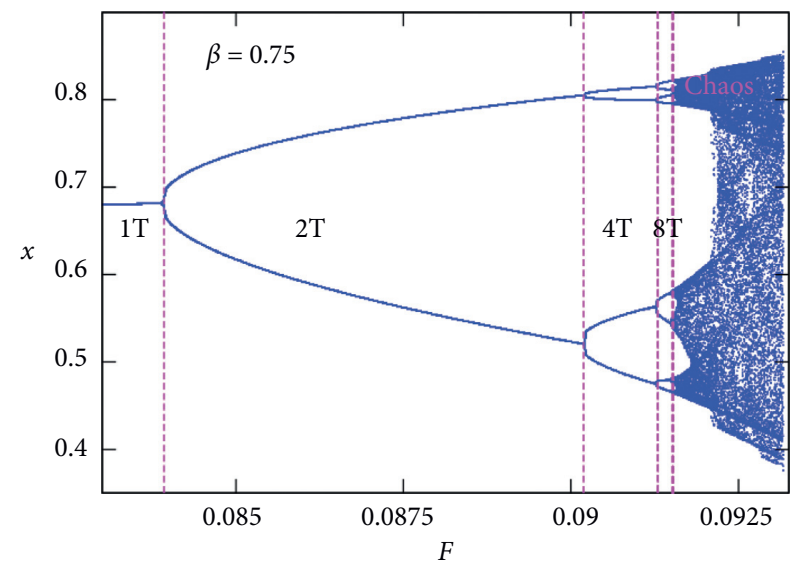

(b)

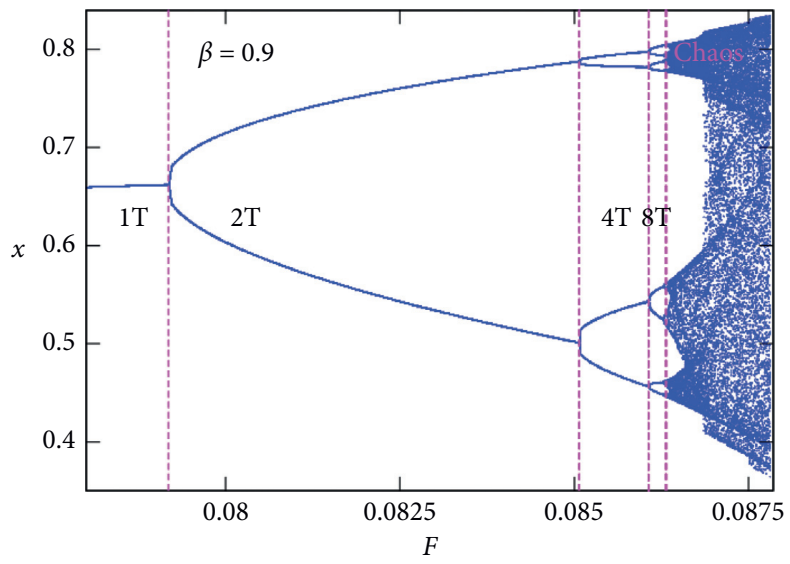

(c)

FIGURE 13: Effect of parameter $\beta$ on the bifurcation diagram of a quadratic-damping Helmholtz oscillator versus $F$ with $\mu=0.1$ and $\Omega=0.85$.

$$
\begin{aligned}
& \frac{\mathrm{d} a}{\mathrm{~d} T_{1}}+\frac{1}{2} \mu a+\left(-B^{2}+\beta B^{2} \Omega^{2}+\nu B^{2} \Omega\right) \sin \phi=0, \\
& a \frac{\mathrm{d} \phi}{\mathrm{d} T_{1}}-\sigma a+\left(-B^{2}+\beta B^{2} \Omega^{2}+\nu B^{2} \Omega\right) \cos \phi=0,
\end{aligned}
$$

where $\phi=\sigma T_{1}-\theta$. When looking for solutions of stationary state, the equation of the superharmonic resonance is

$$
\left(\frac{1}{2} \mu a\right)^{2}+(\sigma a)^{2}=\left[\left(-1+\beta \Omega^{2}+\nu \Omega\right) B^{2}\right]^{2} .
$$

Now, we study the stability of the resonance amplitudes by posing $a=a_{0}+a_{1}$ and $\phi=\phi_{0}+\phi_{1}$, where $\left(a_{0}, \phi_{0}\right)$ is the nontrivial solution and $\left(a_{1}, \phi_{1}\right)$ is supposed infinite perturbations; we obtain

$$
\begin{array}{r}
a_{1}^{\prime}=-\frac{1}{2} \mu a_{1}-P \phi_{1} \cos \phi_{0}, \\
\phi_{1}^{\prime}=\frac{P}{2 a_{0}^{2}} a_{1} \cos \phi_{0}+\frac{P}{a_{0}} \phi_{1} \sin \phi_{0},
\end{array}
$$

where $P=-B^{2}+\beta B^{2} \Omega^{2}+\nu B^{2} \Omega$.
By taking the characteristic equation of the Jacobian matrix of the previous system and then using the Routh-Hurwitz criterion, we conclude that the amplitudes of oscillations are stable if $\mu>0$. Figure 5 represents the effects of the parameters $F$ (see Figure 5(a)), $\beta$ (see Figure 5(b)), and $v$ (see Figure 5(c)) on the superharmonic resonance obtained for $\mu>0$.

Whatever the values of these parameters, we effectively note that the amplitudes of the oscillations are stable, and the superharmonic resonance of order 2 is stable because the amplitudes of resonance correspond to the same frequency of resonance. In addition, we notice that the amplitude of resonance increases with the parameters $F$ and $\delta$, while it decreases with the parameters $\beta$ and $\nu$ of quadratic depreciation. Precisely, the superharmonic resonance of order 2 is accentuated when $F$ and $\delta$ increase and disappears with $\beta$ and $\nu$.

\section{Horseshoe Chaos}

In this section, we analytically determine Smale's horseshoe chaos by Melnikov's method, and then we verify the results numerically by representing the basin of attraction of the 


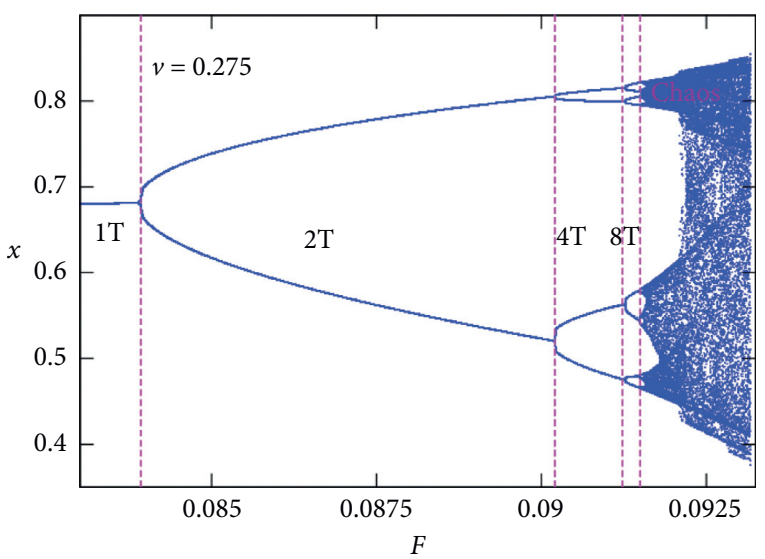

(a)

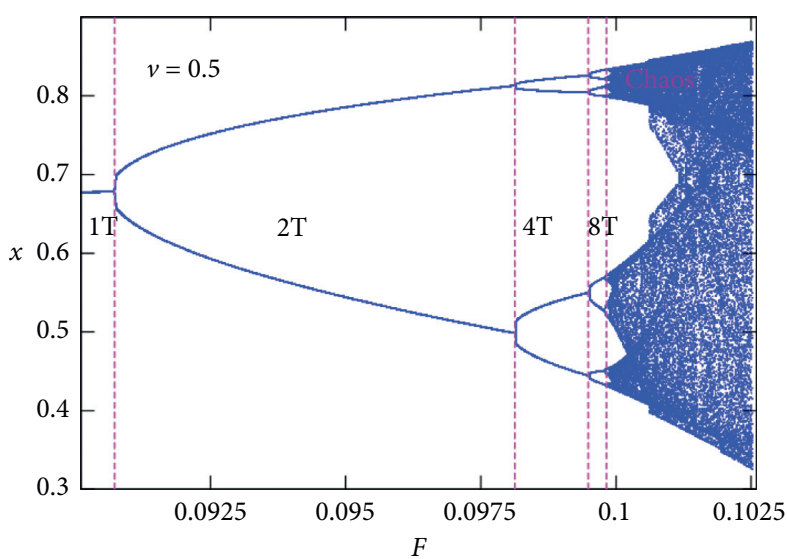

(b)

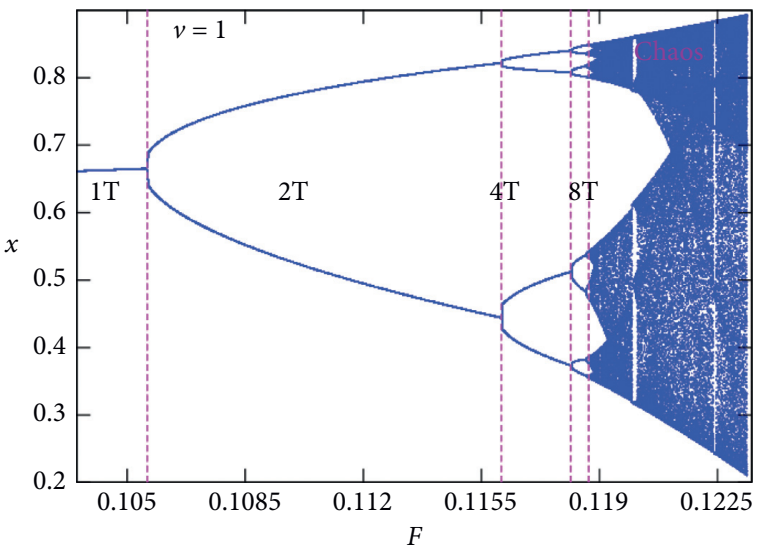

(c)

FIGURE 14: Effect of parameter $\nu$ on the bifurcation diagram of a quadratic-damping Helmholtz oscillator versus $F$ with $\mu=0.1$ and $\Omega=0.85$.

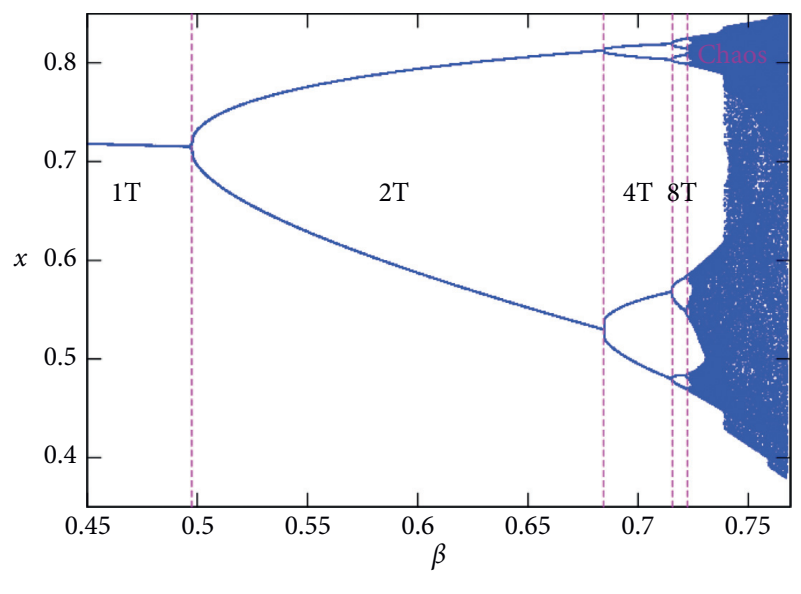

(a)

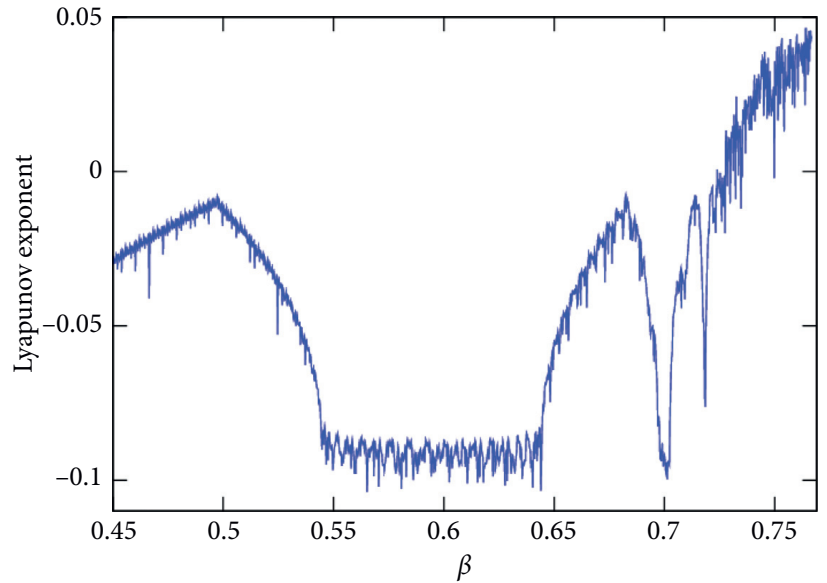

(b)

FIGURE 15: Bifurcation diagram and its corresponding Lyapunov exponent curve of a quadratic-damping Helmholtz oscillator versus $\beta$ with $\mu=0.01, \Omega=0.85, \nu=0.275$, and $F=0.0925$.

system. The unperturbed system of equation (8) can be written as follows:

$$
\begin{array}{r}
\dot{x}=y, \\
\dot{y}=x-x^{2} .
\end{array}
$$




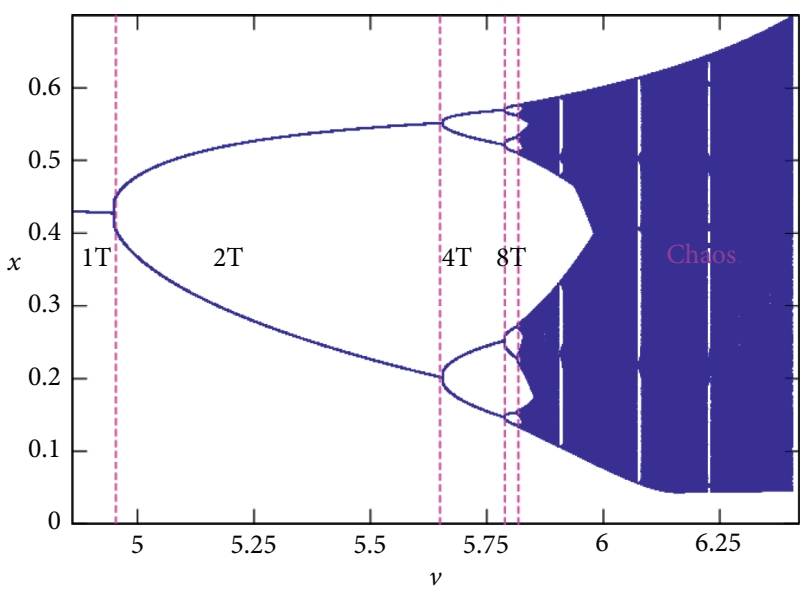

(a)

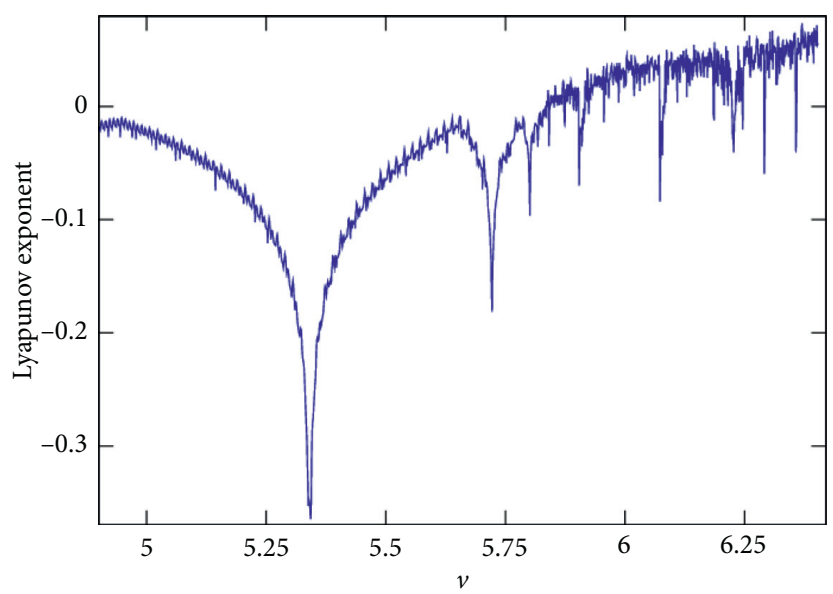

(b)

FIGURE 16: Bifurcation diagram and its corresponding Lyapunov exponent curve of a quadratic-damping Helmholtz oscillator versus $\nu$ with $\mu=0.1, \Omega=0.85, \beta=0.75$, and $F=0.0925$.

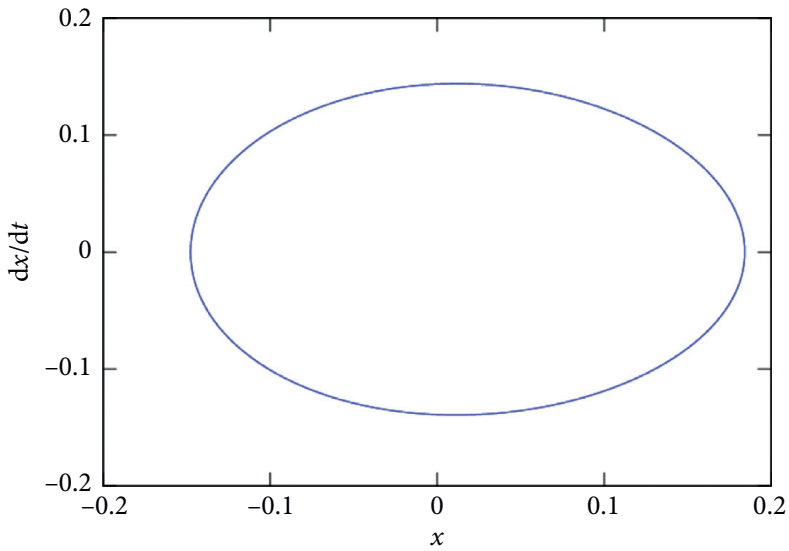

(a)

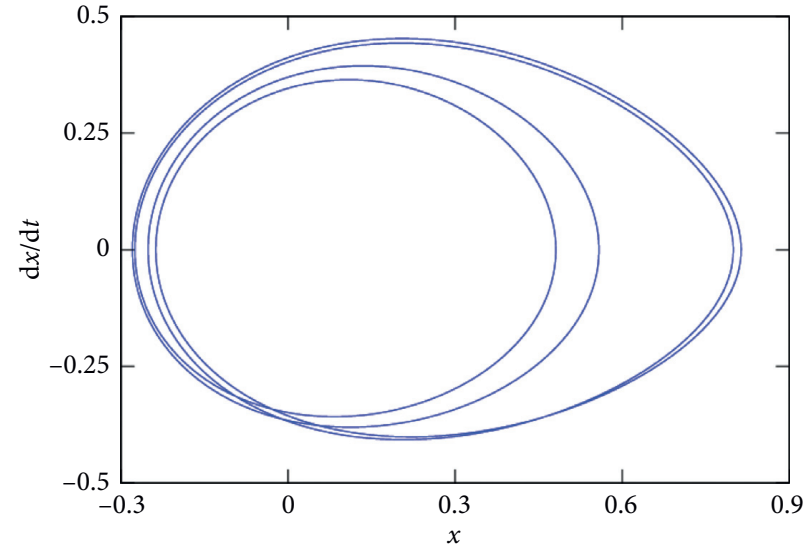

(b)

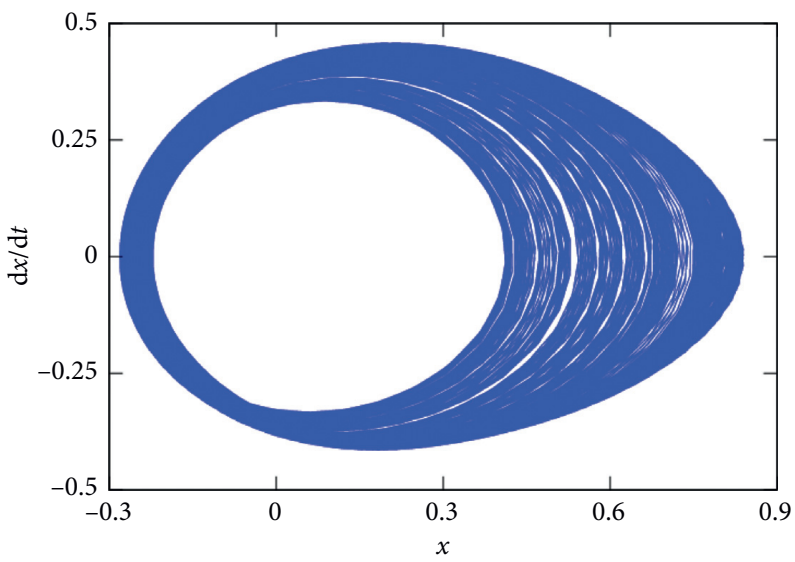

(c)

Figure 17: Various phase portraits with parameters of Figure 13(b): (a) $F=0.0425$; (b) $F=0.091$; (c) $F=0.0925$.

The potential of the system is defined by

$$
V(x)=\frac{1}{2} x^{2}-\frac{1}{3} x^{3}
$$




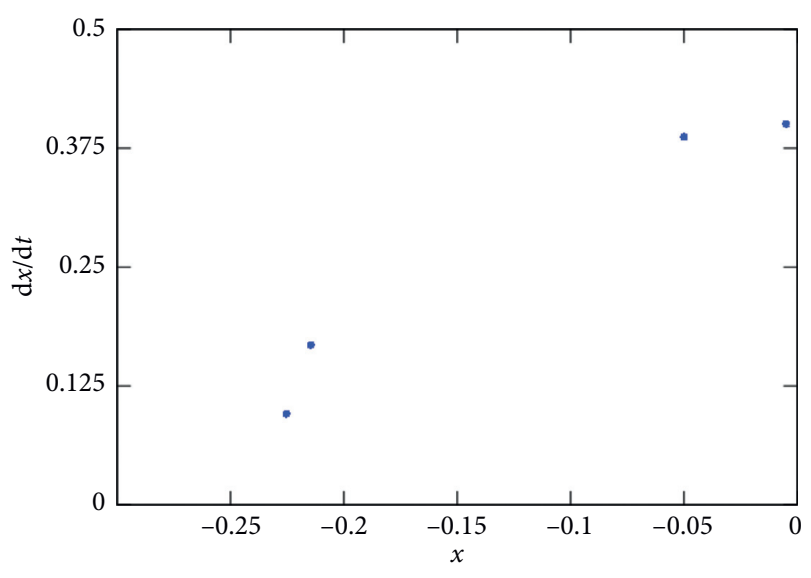

(a)

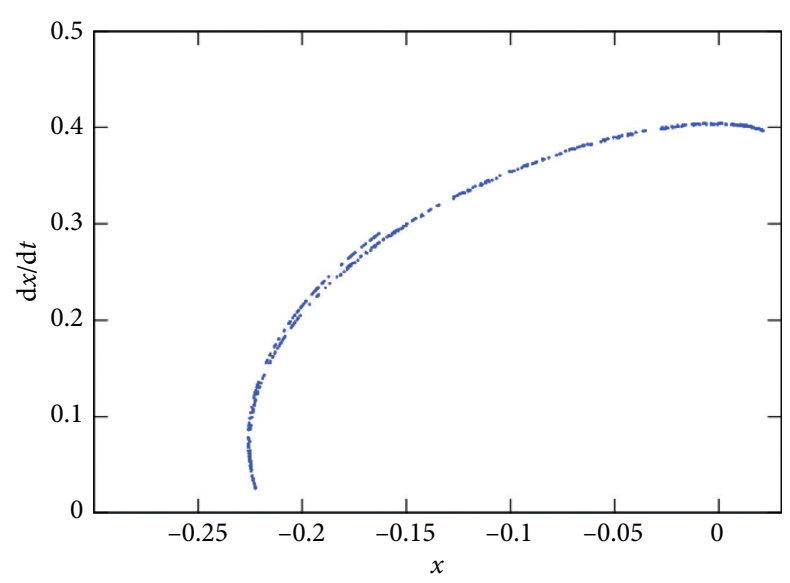

(b)

Figure 18: Poincaré section with parameters of Figure 13(b): (a) $F=0.091$; (b) $F=0.0925$.

The unperturbed system is Hamiltonian, and associated Hamiltonian is

$$
H(x, y)=\frac{1}{2} y^{2}+\frac{1}{2} x^{2}-\frac{1}{3} x^{3}
$$

The homoclinical orbits which connect the fixed points of undisturbed system (38) correspond to zero Hamiltonian. By solving the equation $H(x ; y)=0$, we get

$$
\begin{gathered}
x_{h}=1-\frac{3}{1+\cosh \tau}, \\
y_{h}=\frac{3 \sinh \tau}{[1+\cosh \tau]^{2}} .
\end{gathered}
$$

Figure 6 presents the potential (Figure 6(a)) and homoclinic orbits (Figure 6(b)). It is noted that the potential is a single well and a single hump.

To determine the Melnikov criterion, we put equation (8) in the form

$$
\begin{array}{r}
\dot{x}=y, \\
\dot{y}=F \cos \Omega \tau-x+x^{2}-\mu y+\beta y^{2}-v x y .
\end{array}
$$

By definition, the integral of Melnikov is given by [21-25]

$$
M\left(\tau_{0}\right)=\int_{-\infty}^{+\infty} f\left(x_{h}, y_{h}\right) \wedge g\left(x_{h}, y_{h}\right) \mathrm{d} t
$$

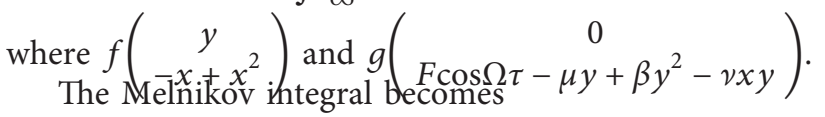

$$
M\left(\tau_{0}\right)=\int_{-\infty}^{+\infty}\left(F y_{h} \cos \Omega \tau-\mu y_{h}^{2}+\beta y_{h}^{3}-v x_{h} y_{h}^{2}\right) \mathrm{d} \tau .
$$

Replacing equation (41) in equation (44), we compute the integral of Melnikov, and we get

$$
M\left(\tau_{0}\right)=-\frac{6 \pi \Omega^{2} F}{\sinh (\Omega \pi)} \sin \left(\Omega \tau_{0}\right)-\frac{6 \mu}{5}-\frac{6 \nu}{35} .
$$

If $M\left(\tau_{0}\right)=0$ and $\mathrm{d} M / \mathrm{d} \tau_{0} \neq 0$ for some $\tau_{0}$ and some sets of parameters, then horseshoes exist, and chaos occurs [21-25]. Using this Melnikov criterion for the appearance of the intersection between the perturbed and unperturbed separatrixes, it is found that chaos appears when the following condition is satisfied:

$$
F \geq F_{\text {cr }}=\left|\frac{B}{A}\right|,
$$

where $A=6 \pi \Omega^{2} / \sin h(\Omega \pi)$ and $B=-(6 \mu / 5+6 \nu / 35)$.

The critical value of $v$ for which Melnikov's chaos appears is

$$
\nu_{c r}=\left|-7 \mu-\frac{35 \pi \Omega^{2} F}{\sin h(\Omega \pi)}\right|
$$

Figure 7 represents the variation of the amplitude of the external excitation according to the frequency. In this figure, the domain located below the curve $\left(F_{\mathrm{cr}}, \Omega\right)$ indicates the domain where the Helmholtz oscillator with quadratic damping has a regular behavior, and above this curve, the oscillator has a chaotic behavior. From the analysis of Figure 7, it appears that the domain of the existence of chaotic behavior of the oscillator studied decreases with $\mu$ and $\nu$. Precisely, the critical value $F_{\mathrm{cr}}$ for the appearance of Smale's horseshoe chaos increases with $\mu$ and $\nu$. For example, with $\Omega=0.297$ and Figure $7(\mathrm{a})$, the chaotic domain corresponds to $F_{\mathrm{cr}}=0.2, F_{\mathrm{cr}}=0.34$, and $F_{\mathrm{cr}}=1.04$ for $\mu=0.275$, $\mu=0.5$, and $\mu=1.75$, respectively, and $F_{\mathrm{cr}}=0.14, F_{\mathrm{cr}}=0.20$, and $F_{\mathrm{cr}}=0.38$ for $\nu=0.75, \nu=3$, and $\nu=5$ (see Figure $7(\mathrm{~b})$ ). Then, we represent in Figure 8 the curve $(\nu \mathrm{cr}, \Omega)$. We observe through this figure that the domain of the existence of chaotic behavior decreases when each of the parameters $\mu$ and $F$ increases. This remark confirms the result of Figure 7(c). In conclusion, the depreciation favors the appearance of the chaos of Melnikov, while the latter is favored by the quadratic force of stiffness. Now, we are going to verify these different results by numerical simulations. Indeed, a better tool to test the validity of the proposed analytical predictions is to investigate numerically the regular 


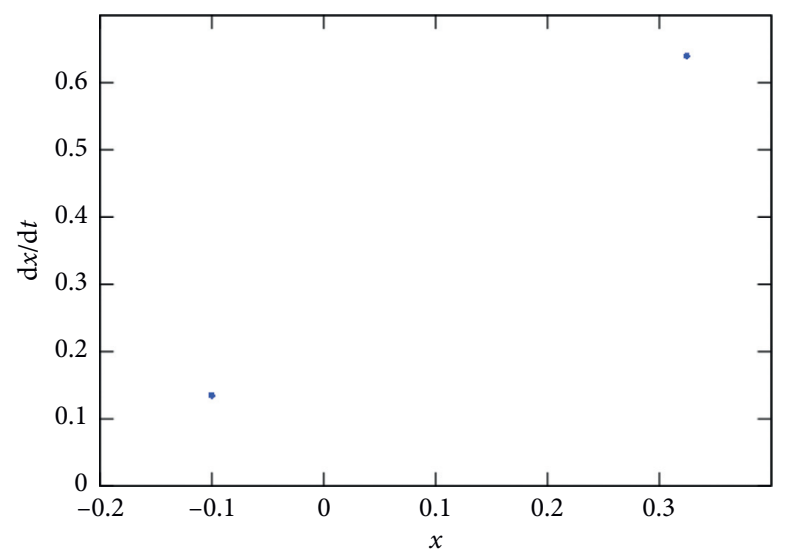

(a)

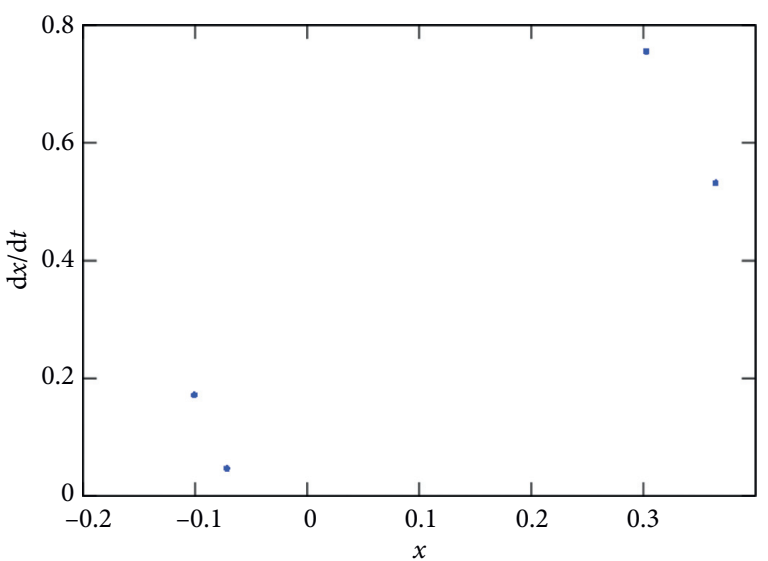

(b)

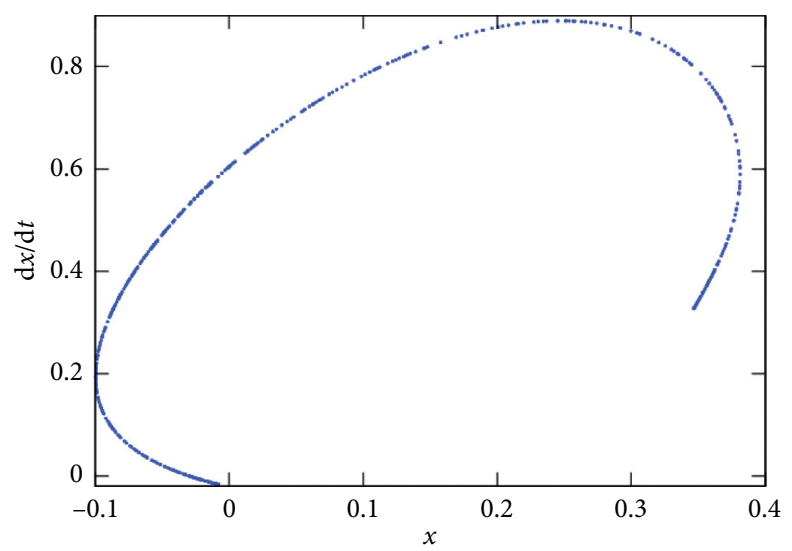

(c)

Figure 19: Effect of $v$ on the Poincaré section with parameters of Figure 16: (a) $v=5.5$; (b) $v=5.75$; (c) $v=6$.

and irregular (fractal) shape of the basins of attraction. The basins are plotted by scanning the initial values $x_{0}$ and $y_{0}$ of $x$ and $y$ domains, solving numerically differential equation (42) and collecting the initial conditions which attracted the dynamics in the single well of the potential. From the Melnikov critical curve (Figure 7), the results obtained after the numerical simulations are recorded in Figures 9-11, respectively, which denote the effect of $F, \mu$, and $\nu$. From these different figures, we note that Melnikov's critical value from which Smale's horseshoe chaos appear is confirmed and also that the effects of $\mu$ and $\nu$ are confirmed. For example, for $F<F_{\mathrm{cr}}$, the basin of attraction of the system is smooth thus showing the regular behavior (Figure 9(a)), while for $F>F_{\text {cr }}$, the basin becomes eroded (Figures 9(b) and 9 (c)) thus proving the presence of Smale's horseshoe chaos. From Figure 10, we observe that the Melnikov critical value increases with $\mu$, and the limits of the basin of attraction become more and more smooth. Finally, we have the same remark for the effect of $\nu$.

\section{Bifurcation and Transition to Chaos}

In this section, we do numerical simulations to look for transition to dissipative chaos. To do this, the bifurcation diagram, the Lyapunov exponent, the phase portrait, and the
Poincaré section are plotted by a direct integration of equation (8) using the fourth-order Runge-Kutta algorithm. Indeed, we analyze the dynamics of the system by taking $\nu=0, \mu=0$, and $\omega=0.85$ (these values are the basic values of the parameters used in the literature [9-14]) with the initial condition $\left(x_{0}=0.2, y_{0}=0.2\right)$, and the results obtained are plotted in Figure 12. From this figure, showing a perfect agreement between the bifurcation diagram and its corresponding Lyapunov exponent, we note that the system oscillates when $0 \leq F \leq 0.109$ with rich dynamics. More precisely, if $0 \leq F \leq 0.10085$, the oscillations of the system are periodical, $1 \mathrm{~T}$; for $0.10085<F \leq 0.1075$, there is a doubling of period, and the oscillations become of period $-2 \mathrm{~T}$. From $0.1075<F \leq 0.1084$, the dynamics of the system become of period $-4 \mathrm{~T}$ and thereafter pass a behavior period $-\mathrm{nT}$ and then become chaotic when $0.10882 \leq F \leq 0.109$. Figures 13 and 14 represent the effects of the pure quadratic parameter $\beta$ and hybrid quadratic parameter $\nu$ of damping, respectively, on the dynamics of the system. Two important observations on the domain of oscillations, in general, and on the domain of chaotic behavior, in particular, are to emerge from the analysis of figures.

Precisely, we observe a phenomenon of successive doubling of period leading to chaos, different domains of which are indicated on the bifurcation diagrams. In other 
words, the system passes successively from oscillations of period $1 \mathrm{~T}, 2 \mathrm{~T}, 4 \mathrm{~T}, 8 \mathrm{~T}$, and $n \mathrm{~T}$ to chaotic oscillations. Indeed, increasing the parameter $\beta$ reduces the oscillation domain of the system but does not have a perceptible effect on the chaotic behavior of the latter (Figure 13).

From Figure 14, we note that the domain of oscillation of the system and that of chaotic behavior increase with the parameter $\nu$. We can therefore note that the parameters $\beta$ and $\nu$ do not act on the system in the same way, and we can therefore use them to control the oscillation domain of the modified Helmholtz oscillator studied and the domain in which the latter possesses chaotic behavior. Finally, Figures 15 and 16 represent the bifurcation diagram and its corresponding Lyapunov exponent with $\beta$ and $\nu$ as control parameters, respectively. These figures show a perfect agreement between the two diagrams and prove that the chaotic behavior is actually favored by the parameter $\nu$. By taking values of $F$ in the appropriate values of each of the behaviors predicted by the bifurcation diagram in Figure 13(b), we obtained the phase portraits and the Poincaré sections of Figures 17 and 18, respectively. We note that the system has a periodic behavior of period $-1 \mathrm{~T}$ for $F=0.0425$, a multiperiodic behavior of period $-4 \mathrm{~T}$ for $F=0.091$, and chaotic if $F=0.0925$. These observations confirm very well the results of Figure 13. Figure 19 represents the Poincaré section of the system for values of $v$ chosen in different domains of behaviors given by Figure 16. We observe at this level the perfect confirmation of the periodic, multiperiodic, and chaotic behaviors of Figure 16 but also of the effect of the hybrid quadratic damping parameter on the dynamics of the modified Helmholtz oscillator.

\section{Conclusions}

In this work, we have studied the quadratic-damping Helmholtz oscillator which models the simple Lotka-Volterra system. The determination of the equilibrium points of the oscillator results in the latter having two fixed points, one of which may be a node or a stable focus or unstable, while the second is a saddle point. The harmonic, primary, and secondary second-order resonances are obtained from the results of treatments by the methods of harmonic balance and multiple scales. The phenomena of amplitude jump and hysteresis are also obtained. It appears that the system has two or three amplitudes for the same value of $F$ or which do not have the same stability. This makes the behavior of the oscillator complex and gives it interesting properties and properties that can be used in memory systems. Subsequently, the Melnikov criterion is used to analytically determine Smale's horseshoe chaos. The basins of attraction are used to numerically verify the results obtained, and there is a perfect confirmation of the prediction given by the Melnikov technique. Knowing that the presence of Smale's horseshoe chaos does not exclude the presence of the dissipative chaos, we used bifurcation diagrams, Lyapunov exponents, phase portraits, and the Poincaré section to detect the road to dissipative chaos in the modified Helmholtz oscillator. It appears that the system studied can present very rich and varied behaviors such as periodic, double-periodic, multiperiodic, and chaotic behaviors. Finally, by analyzing numerically the effects of all the parameters of the system, in general, and, in particular, those of the new parameters, we have the domains of these parameters for which the oscillator vibrates and the domains of interesting behaviors. We noted that all the parameters of the system influence the frequencies and amplitudes of oscillation of the latter in the cases of resonance, amplitude jump, and hysteresis. For the road to chaos, we note that $F$ and $\Omega$ have significant effects on the dynamics of the system, but the parameter of pure quadratic dissipation $\beta$ has a less significant effect on the appearance of chaos for the system studied.

\section{Data Availability}

No data were used in this study.

\section{Conflicts of Interest}

The authors declare that they have no conflicts of interest.

\section{References}

[1] F. Bernardot, J. Bruneaux, and J. Matricon, "Un archétype d'oscillateur: le résonateur acoustique de Helmholtz," Bulletin de l'Union des Physiciens, vol. 96, pp. 1055-2076, 2002.

[2] A. Doelman, A. Femius Koenderink, and L. R. M. Maas, "Quasi-periodically forced nonlinear Helmholtz oscillators," Physica D: Nonlinear Phenomena, vol. 164, no. 1-2, pp. 1-27, 2002.

[3] F. Balibrea, R. Chacón, and M. A. López, "Reshaping-induced order-chaos routes in a damped driven Helmholtz oscillator," Chaos, Solitons \& Fractals, vol. 24, no. 2, pp. 459-470, 2005.

[4] A. I. Komkin and A. I. Bykov, "Inertial attached neck length of Helmholtz resonators," Acoustical Physics, vol. 62, no. 3, pp. 269-279, 2016.

[5] T. Poston and I. N. Stewart, Catastrophe Theory and its Applications (London: Pitman), 1978.

[6] M. E. Goggin and P. W. Milonni, "Driven Morse oscillator: classical chaos, quantum theory, and photodissociation," Physical Review A, vol. 37, no. 3, pp. 796-806, 1988.

[7] R. Chacón and J. I. Cirac, "Chaotic and regular behavior of a trapped ion interacting with a laser field," Physical Review A, vol. 51, no. 6, pp. 4900-4905, 1995.

[8] J. B. Buchler and H. Einchhorn, "Chaotic Phenomena in Astrophysics," Annals of the New York Academy of Sciences, vol. 497, 1987.

[9] J. M. T. Thompson, "Chaotic phenomena triggering the escape from a potential well," Proceedings of the Royal Society of London, vol. 421, pp. 195-225, 1989.

[10] J. M. T. Thompson and M. S. Soliman, "Fractal control boundaries of driven oscillators and their relevance to safe engineering design," in Engineering Applications of Dynamics of Chaos, W. Szemplinska-Stupnicka and H. Troger, Eds., vol. 319, International Centre for Mechanical Sciences (Courses and Lectures), Springer, Vienna, 1991.

[11] M. S. Soliman, "Basin boundaries with fractal and smooth accumulation properties in systems with single potential well," Chaos, Solitons \& Fractals, vol. 9, no. 6, 1998.

[12] R. Irving, J. Epstein, and A. Pojman, An Introduction to Nonlinear Chemical Dynamics: Oscillations, Waves, Patterns 
and Chaos, Oxford University Press, New York, NY, USA, 1998.

[13] J. M. T. Thompson, "Designing against capsize in beam seas: recent advances and new insights," Applied Mechanics Reviews, vol. 50, no. 5, pp. 307-325, 1997.

[14] C. Ainamon, S. T. Kingni, V. K. Tamba, J. B. C. Orou, and P. Woafo, "Dynamics, circuitry implementation and control of an autonomous Helmholtz Jerk oscillator," Journal of Control, Automation and Electrical Systems, vol. 30, no. 4, pp. 501-511, 2019.

[15] K. J. Spirou, B. Cotton, and G. Cotton, "Analytical expressions of capsize boundary of a ship with roll bias in beam waves," Journal of Ship Research, vol. 46, pp. 167-174, 2002.

[16] J. A. Gottwald, L. N. Virgin, and E. H. Dowell, "Routes to escape from an energy well," Journal of Sound and Vibration, vol. 187 , no. 1 , pp. 133-144, 1995.

[17] I. S. Kang and L. G. Leal, "Bubble dynamics in time-periodic straining flows," Journal of Fluid Mechanics, vol. 218, pp. 41-69, 1990.

[18] A. H. Nayfey and D. T. Mook, Nonlinear Oscillations, Wiley, New York, NY, USA, 1979.

[19] D. L. Olabodé, C. H. Miwadinou, V. A. Monwanou, and J. B. Chabi Orou, "Effects of passive hydrodynamics force on harmonic and chaotic oscillations in nonlinear chemical dynamics," Physica D: Nonlinear Phenomena, vol. 386-387, pp. $49-59,2019$.

[20] C. H. Miwadinou, L. A. Hinvi, A. V. Monwanou, and J. B. Chabi Orou, "Nonlinear dynamics of a \$\$Varvec\{\phi 6$\}$ $\$ \$ \phi 6$ - modified Duffing oscillator: resonant oscillations and transition to chaos," Nonlinear Dynamics, vol. 88, no. 1, pp. 97-113, 2017.

[21] M. S. Siewe, H. Cao, and M. A. F. Sanjuán, "On the occurrence of chaos in a parametrically driven extended Rayleigh oscillator with three-well potential," Chaos, Solitons \& Fractals, vol. 41, no. 2, pp. 772-782, 2009.

[22] C. H. Miwadinou, A. V. Monwanou, J. Yovogan, L. A. Hinvi, P. R. Nwagoum Tuwa, and J. B. Chabi Orou, "Modeling nonlinear dissipative chemical dynamics by a forced modified Van der Pol-Duffing oscillator with asymmetric potential: chaotic behaviors predictions," Chinese Journal of Physics, vol. 56, no. 3, pp. 1089-1104, 2018.

[23] C. H. Miwadinou, A. V. Monwanou, L. A. Hinvi, and J. B. Chabi Orou, "Effect of amplitude modulated signal on chaotic motions in a mixed Rayleigh-Liénard oscillator," Chaos, Solitons \& Fractals, vol. 113, pp. 89-101, 2018.

[24] C. H. Miwadinou, A. V. Monwanou, A. A. Koukpemedji, Y. J. F. Kpomahou, and J. B. Chabi Orou, "Chaotic motions in forced mixed Rayleigh -Liénard oscillator with external and parametric periodic-excitations," Journal of Bifurcation and Chaos, vol. 28, no. 3, Article ID 1830005, 2018.

[25] D. L. Olabod_e, C. H. Miwadinou, V. A. Monwanou, and J. B. Chabi Orou, "Horseshoes chaos and its passive control in dissipative nonlinear chemical dynamics," Physica Scripta, vol. 93, 2018.

[26] M. A. F. Sanjuán, "The effect of nonlinear damping on the universal escape oscillator," International Journal of Bifurcation and Chaos, vol. 9, no. 4, pp. 735-744, 1999.

[27] M. S. Soliman and J. M. T. Thompson, "The effect of damping on the steady state and basin bifurcation patterns of a nonlinear mechanical oscillator," International Journal of Bifurcation and Chaos, vol. 2, no. 1, pp. 81-91, 1992.

[28] C. H. Miwadinou, A. V. Monwanou, and J. B. Chabi Orou, "Effect of nonlinear dissipation on the basin boundaries of a driven two-well modified Rayleigh-duffing oscillator,"
International Journal of Bifurcation and Chaos, vol. 25, no. 2, p. 1550024, 2015.

[29] C. H. Miwadinou, A. V. Monwanou, L. A. Hinvi, A. A. Koukpemedji, C. Ainamon, and J. B. Chabi Orou, "Melnikov chaos in a modified Rayleigh-Duffing oscillator with $\varphi^{6}$ potential," International Journal of Bifurcation and Chaos, vol. 26, no. 5, Article ID 1650085, 2016.

[30] M. Amabili, "Derivation of nonlinear damping from viscoelasticity in case of nonlinear vibrations," Nonlinear Dynamics, vol. 97, no. 3, pp. 1785-1797, 2019.

[31] M. Amabili, "Nonlinear damping in nonlinear vibrations of rectangular plates: Derivation from viscoelasticity and experimental validation," Journal of the Mechanics and Physics of Solids, vol. 118, pp. 275-292, 2018.

[32] P. Balasubramanian, G. Ferrari, and M. Amabili, "Identification of the viscoelastic response and nonlinear damping of a rubber plate in nonlinear vibration regime," Mechanical Systems and Signal Processing, vol. 111, pp. 376-398, 2018.

[33] M. Amabili, "Nonlinear damping in large-amplitude vibrations: modelling and experiments," Nonlinear Dynamics, vol. 93, pp. 5-18, 2018.

[34] M. I. Evstifeev, A. S. Kovalev, and D. P. Eliseev, "Electromechanical model of RR-Type MEMS gyro with consideration for the platform vibrations," Gyroscopy and Navigation, vol. 5, no. 3, pp. 174-180, 2014.

[35] Z. Iskakov and K. Bissembayev, "The nonlinear vibrations of a vertical hard gyroscopic rotor with nonlinear characteristics," Mechanical Sciences, vol. 10, no. 2, pp. 529-544, 2019.

[36] R. G. Wang, Y. Chen, and G. W. Cai, "Subharmonic Resonance Analysis of a 2-DOF Controllable Linkage Mechanism System Affected by Self-Excited Inertial Force," Advanced Materials Research, vol. 139-141, pp. 2381-2385, 2010.

[37] C. Hayashi, Nonlinear Oscillations in Physical Systems, McGraw-Hill, New York, NY, USA, 1964. 